\title{
ulti-institutional dosimetric delivery assessment of intracranial stereotactic radiosurgery on different treatment platforms
}

DOI:

10.1016/j.radonc.2020.05.024

\section{Document Version}

Accepted author manuscript

Link to publication record in Manchester Research Explorer

Citation for published version (APA):

Dimitriadis, A., Tsang, Y. M., Thomas, R., Palmer, A., Eaton, D., Lee, J., Patel, R., Silvestre Patallo, I., Gouldstone, C., Snaith, J., Kirkby, K., Nisbet, A., \& Clark, C. (2020). ulti-institutional dosimetric delivery assessment of intracranial stereotactic radiosurgery on different treatment platforms. Radiotherapy \& Oncology, 147, 153-161. https://doi.org/10.1016/j.radonc.2020.05.024

\section{Published in:}

Radiotherapy \& Oncology

\section{Citing this paper}

Please note that where the full-text provided on Manchester Research Explorer is the Author Accepted Manuscript or Proof version this may differ from the final Published version. If citing, it is advised that you check and use the publisher's definitive version.

\section{General rights}

Copyright and moral rights for the publications made accessible in the Research Explorer are retained by the authors and/or other copyright owners and it is a condition of accessing publications that users recognise and abide by the legal requirements associated with these rights.

\section{Takedown policy}

If you believe that this document breaches copyright please refer to the University of Manchester's Takedown Procedures [http://man.ac.uk/04Y6Bo] or contact uml.scholarlycommunications@manchester.ac.uk providing relevant details, so we can investigate your claim.

\section{OPEN ACCESS}


Radiotherapy and Oncology

\section{Multi-institutional dosimetric delivery assessment of intracranial stereotactic radiosurgery on different treatment platforms \\ --Manuscript Draft--}

\begin{tabular}{|c|c|}
\hline Manuscript Number: & RO-D-19-01530R1 \\
\hline Article Type: & Full Length Article \\
\hline Keywords: & $\begin{array}{l}\text { Radiosurgery; Dosimetry; End-to-end; Audit; Anthropomorphic Phantom; Alanine; } \\
\text { Radiochromic film }\end{array}$ \\
\hline Corresponding Author: & $\begin{array}{l}\text { Alexis Dimitriadis, B.Sc., M.Sc., Ph.D. } \\
\text { University of Surrey } \\
\text { Guildford, UNITED KINGDOM }\end{array}$ \\
\hline First Author: & Alexis Dimitriadis, B.Sc., M.Sc., Ph.D. \\
\hline \multirow[t]{13}{*}{ Order of Authors: } & Alexis Dimitriadis, B.Sc., M.Sc., Ph.D. \\
\hline & Yat Man Tsang, PhD \\
\hline & Russell AS Thomas, MSc \\
\hline & Antony L Palmer, PhD \\
\hline & David Eaton, PhD \\
\hline & Jonathan Lee, MSc \\
\hline & Rushil Patel, MSc \\
\hline & Ileana Silvestre Patallo, MSc \\
\hline & Clare Gouldstone, MSc \\
\hline & Julia AD Snaith, PhD \\
\hline & Karen J Kirkby, PhD \\
\hline & Andrew Nisbet, $\mathrm{PhD}$ \\
\hline & Catharine H Clark, PhD \\
\hline Abstract: & $\begin{array}{l}\text { Background and Purpose: } \\
\text { Assessment of dosimetric accuracy of radiosurgery on different treatment platforms. } \\
\text { Material and Methods: } \\
\text { Thirty-three single fraction treatment plans were assessed at thirty centres using an } \\
\text { anthropomorphic head phantom with target and brainstem structures. The target being } \\
\text { a single irregular shaped target, } ~ 8 c c, 10 \mathrm{~mm} \text { from the brainstem. The phantom was } \\
\text { "immobilised", scanned, planned and treated following the local protocols. EBT-XD } \\
\text { films and alanine pellets were used to measure absolute dose, inside both the target } \\
\text { and the brainstem, and compared with TPS predicted dose distributions. } \\
\text { Results: } \\
\text { PTV alanine measurements from gantry-based linacs showed a median percentage } \\
\text { difference to the TPS of } 0.65 \% \text {. Cyberknife (CK) had the highest median difference of } \\
2.3 \% \text { in comparison to the other platforms. Gamma Knife (GK) showed the smallest } \\
\text { median of } 0.3 \% \text {. Similar trends were observed in the OAR with alanine measurements } \\
\text { showing median percentage differences of } 1.1 \%, 2.0 \% \text { and } 0.4 \% \text {, for gantry-based } \\
\text { linacs, CK and GK respectively. All platforms showed comparable gamma passing } \\
\text { rates between axial and sagittal films. } \\
\text { Conclusions: } \\
\text { This comparison has highlighted the dosimetric variation between measured and TPS } \\
\text { calculated dose for each delivery platform. The results suggest that clinically } \\
\text { acceptable agreement with the predicted dose distributions is achievable by all } \\
\text { treatment delivery systems. }\end{array}$ \\
\hline
\end{tabular}




\section{Covering Letter}

Dear editor,

This is a resubmission of manuscript RO-D-18-00727 that was previously rejected with major corrections.

We previously received very good comments from all four reviewers on the importance of this work. We believe that with the revisions done, we have addressed the comments of the reviewers and this is now a unique study which addresses a gap in the literature and will generate several citations in the future.

We look forward to receiving your feedback.

Best Regards,

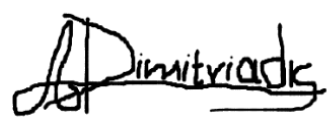

Alexis Dimitriadis

Authors: Alexis Dimitriadis ${ }^{1,2,5} \mathrm{PhD}^{*}$, Yatman Tsang ${ }^{4} \mathrm{PhD}$, Russell A.S. Thomas ${ }^{2} \mathrm{MSc}$, Antony L. Palmer ${ }^{1,3} \mathrm{PhD}$, David Eaton ${ }^{4} \mathrm{PhD}$, Jonathan Lee ${ }^{4} \mathrm{MSc}$, Rushil Patel ${ }^{4} \mathrm{MSc}$, lleana Silvestre Patallo ${ }^{2}$ MSc, Clare Gouldstone ${ }^{2}$ MSc, Julia A.D. Snaith ${ }^{2}$ MSc, Karen J. Kirkby ${ }^{6,7} \mathrm{PhD}$, Andrew Nisbet ${ }^{2,8} \mathrm{PhD}$, Catharine H. Clark ${ }^{1,2,4,5} \mathrm{PhD}$

1. Faculty of Engineering and Physical Sciences, University of Surrey, Guildford, Surrey, UK

2. Radiation Dosimetry Group, National Physical Laboratory, Teddington, Middlesex, UK

3. Medical Physics Department, Portsmouth Hospitals NHS Trust, Portsmouth, UK

4. Radiotherapy Trials Quality Assurance Group, Mount Vernon Hospital, London, UK

5. Department of Medical Physics, Royal Surrey County Hospital NHS Foundation Trust, Guildford, Surrey, UK

6. The Christie NHS Foundation Trust, Manchester, UK

7. Institute of Cancer Sciences, University of Manchester, Manchester, UK

8. University College London, London, UK

*Corresponding author: dimitriadis.alexis@gmail.com 


\section{Highlights:}

- A multi-institutional end-to-end assessment of radiosurgery dosimetric delivery was performed for 33 plans in 30 participating centres using a variety of treatment platforms.

- The comparison has highlighted the dosimetric consistency achievable with different delivery platforms.

- The need for standardisation in intracranial stereotactic radiosurgery is highlighted. 
Dear Editor,

We would like to thank the reviewers for their detailed and comprehensive reviews. We believe that in addressing the reviewers' concerns we have further improved the paper and hope that it is now suitable for publication.

Reviewer \#1: The manuscript is concerned with a dosimetric intercomparison of stereotactic intracranial radiotherapy in UK with particular emphasis on the performance of different technologies. As these procedures are becoming more popular this is a relevant topic of interest to readers of the Green Journal.

Response: We thank the reviewer for this comment and agree that this is a relevant topic of interest to readers of the Green Journal.

It would be important to clarify if cones, tertiary microMLCs or other accessories were used for the linac based deliveries. I also do not think tomotherapy with a fixed ring gantry and no non-coplanar deliveries should be combined in with other linac based deliveries.

Response: The grouping of the technological platforms has been based upon their broadly similar dosimetric and clinical practices (ref 6) and this justification has now been emphasised within the manuscript and reference made to the use of cones, tertiary microMLCs etc.

Maybe it would also be worthwhile to directly compare FFF and flattened delivery systems directly.

Was an attempt made to correlate different metrices from individual centres (eg is a centre that has difficulties getting high gammas in the film comparison also a larger chance to have a larger alanine output measurement difference?

Response: No attempt has been made within this study to directly compare FFF and flattened delivery systems directly. Each centre received a full dosimetric report of their output measurement difference and gamma analysis of their film comparison but no correlation between these parameters has been performed.

It should also be checked if the figures and table are correctly labeled as they refer to 33 centres while the manuscript refers to 30 centres with 33 treatment platforms. As there may be some common features even of different technologies in a single centre it would be better to show which centres utilize more than one technology (unless this would identify the centres in an UK setting but this may already be possible also from other technical details, eg only one tomotherapy unit).

Response: Centres has been replaced by "platform number" in the respective tables and figures. To avoid identifying individual centres those centres which utilise more than one technology have not been identified.

Specific comments:

Highlights: the third highlight appears to be a bit optimistic - would 'centre' 16's

Response: The third highlight has been deleted 
Abstract: It would be more informative if range of difference was given as $-\ldots+\ldots$ instead of a single number.

Response: the reporting of ranges has been deleted in the abstract

Line 147: please provide manufacturer and location for QAPro.

Response: These details (Ashland ISP Advanced Materials, NJ, USA) are within the text

Line 163: maybe add AAPM to TG

Response: AAPM has been added.

Results, para1: Given the fact that the number of tests for different pieces of equipment was different, the 'spread' is not necessarily a meaningful quantity to compare as more measurements are likely to create a greater spread even if the standard deviation is identical.

Response: the reporting of ranges within each subgroup has been maintained as this provides information on the consistency in dosimetry between centres using these platforms, however the comparison of the range between different subgroups has been deleted.

Line 250: also tomotherapy has single energy

Response: the text has been changed to acknowledge this

Table 1: As the authors rightly point out the prescription details are one of the more interesting features of the manuscript. One of the last two columns could be omitted though as they show identical information.

Response: the last column has been deleted.

Figures: while there is an attempt made to use the same symbols throughout, the formatting of figures 3, 4, 5 and A2 could be more uniform (aspect ratio, location of legend)

Response: the formatting has been edited to create a more uniform appearance. 
Reviewer \#2: I have previously reviewed this work back in 2018 and found a major revision necessary before publication of the manuscript. After re-reading the work now and my initial review and criticism I find that all points previously raised have been well addressed such that I can now vote this important work for publication as is. I have no major or minor comments left, but, at the authors discretion, to add some more results detailed (e.g., on the film evaluation) in the abstract.

Response: we would like to thank the reviewer for recommending this "important work" for publication.

Reviewer \#3: The authors have addressed most of the reviewer's comments from the previous version. The statistical analysis has been performed and this has clearly improved the soundness of the discussion section.

Response: We would like to thank the reviewer for his comments on the improved soundness of the discussion section.

However there are still minor issues that would merit some consideration:

Why didn't the auditors perform a reference dose measurement using alanine pellets?

Response: the reference dose was measured according to the appropriate dosimetry code of practice using the ionisation chamber recommended there. However, alanine were employed to measure absolute dose in the target and brainstem.

It would be of interest to know how many institutions using gantry based linacs used, VMAT arcs or 3DCRT (coplanar or non coplanar)

Response: This information is given in Table 1.

L68 I would suggest to use "assess" instead of "determine"

Response: The wording has been changed as suggested

L98 the collimation systems should be added to table 1. If too busy, it should, at least, be mentioned that CK used cones and not iris collimator.

Response: it has been mentioned that CK used cones.

L111 Was the geometric phantom "water" or was it forced to match the phantom material?

Response: The geometric phantom was water equivalent. This has been clarified in the text

L117 Could you state the dimensions of the alanine pellets?

Response: The pellets were discs each of $5 \mathrm{~mm}$ diameter and $2.5 \mathrm{~mm}$ thickness. This has been added to the text. 
L144 State that the films were placed inside the phantom

Response: This wording has been added

L177 As in last version, this sentence is confusing. Did the centres performed reference output measurements? There is a need for clarification. Although in methods is not said figure 2 clearly states that measurements are performed by the centres. If so the used detector could be of interest to the readers and also could be used for the discussion on discrepancies.

Response: This sentence has been clarified to read "Reference output measurements were performed independently by both the host centre and the auditors using their respective equipment and these are presented in Figure 3." No analysis has been made based on the host centres' detectors

L197 Does this apply for all platforms, please state.

Response: This has been reworded as: "Individual pellet measurements in the target showed differences of up to $14.1 \%, 4.2$ and $5.9 \%$ (for GK, CK, linac respectively) when compared to the TPS-predicted mean dose in their individual contoured pellet structure."

L255 I don't see any problem for gantry based linacs where the reference field is $10 \times 10 \mathrm{~cm} 2$. Even by using FFF the KQ,Q0 factors in TRS483 correct for this averaging.

Response: This work predates the publication of TRS-483 and was not taken into account at the time the study was conducted.

L274 Not in the accuracy of the delivery but in the dose calculation accuracy.

Response: This has been corrected.

L333 Shouldn't conclusion start here?

Response: The conclusion has been started at this point.

L351 "approach"?

Response: This has been corrected

L379 Although I fully agree, I still don't know how this is demonstrated by your study.

Response: This sentence has been rephrased to read "Future multicentre SRS studies may benefit from some standardisation and consensus of practice." 
Reviewer \#4: I have major concerns about the way the results have been presented (also relevant to discussion, conclusion, etc). The data is being evaluated as if it was a singlevariable dataset with variations attributed solely to the delivery platform. le., delivery is from a gammaknife, a cyberknife, or linac-based system (which includes tomotherapy). This ignores the other variables and confounders that exist in the dataset. A large one is the dose calculation platform and algorithm, of which there are numerous - it seems probable to me that this has a bigger impact on the dose accuracy (and range of accuracy) than the delivery platform, but it is essentially ignored in the analysis. I would also expect the immobilization to play a role - dose gradients are steep enough that misalignments will impact the dose agreement with the TPS. Although this issue is raised briefly in the discussion, I think the issue is bigger - the results are not reasonable to presented because the groups are not really groups as they have been lumped by a single common parameter.

Response: Dosimetry intercomparisons have an acknowledged part to play in confirming the consistency in dose delivery for advanced radiotherapy treatments. This is the first reported study investigating intracranial stereotactic radiosurgery and is therefore a valuable addition to the literature. In the sense that all centres were delivering intracranial stereotactic radiosurgery this is a single dataset. The methodologies for grouping within that dataset are multiple. However, for the results to be presented in a meaningful manner it is important that the subsets within this single dataset are grouped such as to enable adequate numbers to enable some statistical calculation of significance. The justification for the groupings considered has been emphasised within the text. It is acknowledged that this audit has come from a wide range of delivery and ancillary systems, however it has the potential to benchmark what can be achieved with intracranial stereotactic surgery systems and therefore contribute data for use in setting tolerances for clinical trials and external audits.

Ln 181-186. If there weren't any statistical differences, then you can't say that the linac group had the largest spread or that the GK had the smallest spread. This is the whole point of statistical analysis and the implications of the results. Although I'm also concerned because your description is focused substantially on which platform has the largest range of values, but your methods only talks about a Kruskal-Wallis test (and associated post hoc analysis) which is not appropriate for comparing ranges or variances, only means. You need a Levene test of Variance or something like that to evaluate the relative spreads.

Response: the reporting of ranges within each subgroup has been maintained as this provides information on the consistency in dosimetry between centres using these platforms, however the comparison of the range between different subgroups has been deleted.

Ln 191-194. Similar problem. The spread for linacs is qualified as being "larger". There is not evaluation to support this. You have observational data here (for the ranges) and no qualification is appropriate without actual evaluation. Simply state that the range was "X", or, better yet, actually evaluate the data to see if there is a difference.

Ultimately, compared to the number of variables that you have in play here, you really have 33 distinct anecdotes rather than data in groups that can be compared. I think there is still substantial value in what was found, but I do not think you can draw the results and conclusions, as presented, are valid.

Response: As in the previous reply the reporting of ranges within each subgroup has been maintained as this provides information on the consistency in dosimetry between centres using these platforms, however the comparison of the range between different subgroups has been deleted. 
Minor

Ln 124. I do not understand how comparing the mean TPS and alanine doses allowed you to account for positional uncertainties. Are you implying that you excluded specific results if they didn't agree but the mean did?

Response: Small positional errors in set up of the alanine pellets can result in large differences between measured and calculate dose in areas of large dose gradients. By employing mean doses this takes the dose over a larger area and so effectively smooths out any positional errors. No specific results were excluded.

Ln 126. I can't figure out how you "normalize" a percent difference. Please clarify.

Response: this may be explained as follows:

[(DOSE_alanine/12Gy)-(DOSE_tps/12Gy)]*100\%

Ln 154. The optimum shift algorithm is relevant for small shifts. This indicates a different approach was used to align to high precision to begin with (at least within $1 \mathrm{~mm}$ ). What was this initial alignment method?

Response: An additional explanation has been added to the text stating:

"The measured dose distributions were matched to TPS dose distributions by aligning the fiducial marker holes in the film with known coordinates in the TPS dose planes and then using the optimum shift algorithm, where small shifts were applied $\left(<1 \mathrm{~mm}\right.$ and $\left.<1^{\circ}\right)$ to optimise the gamma passing rate".

159 "the criteria selected were based on their clinical significance". Yet the authors used $3 \% / 2 \mathrm{~mm}$ because its common, $2 \% / 2 \mathrm{~mm}$ because of TG-135, and then include $5 \% / 1 \mathrm{~mm}$ as being more suitable. None of these appear to be based on clinical significance. Please provide relevant citations indicating the clinical impact in SRS of these (and for $5 \% / 1 \mathrm{~mm}$ being more suitable), or more easily, just state that you "selected the following criteria for evaluation....."

Response: As suggested we have now stated that the criteria were selected as follows

170 "Kruskai" should be "Kruskal"

Response: This has been corrected

Supplemental figure 1. I don't think this is necessary.

Response: As a supplementary figure we are happy for this to be deleted and leave the final decision with the editor. 
Ln 132. "passing rates improved significantly". Please provide magnitude and associated $p$ values (or at least max $p$ value over the cohort).

Response: This has been rephrased to: "passing rates improved substantially".

Ln 245. Why would a smaller number of centres lead to more consistent outputs?

Response: this has been deleted

Ln 308-310. I would think that the steeper gradient of the GK (and to a lesser extent the GK) would be an important factor in elevating the gamma pass rate because dose criteria would be easiest to meet under conditions of steeper gradients.

Response: This sentence has been reworded to emphasise the impact of the steeper GK dose gradients.

Ln 333-366 consider shortening or removing as they are not incorrect, but the first two paragraphs feel substantially unnecessary, the third is dramatically away from the findings of the current study.

Response: The first and second paragraphs have been shortened to clarify and the third paragraph has been removed. The concluding sentence has been rephrased to better reflect the findings of the study. 
Short title: Multi-institutional audit of dosimetric delivery in radiosurgery

1 Title: Multi-institutional dosimetric delivery assessment of intracranial stereotactic radiosurgery on different treatment platforms

Keywords: Radiosurgery, Dosimetry, End-to-end, Audit, Anthropomorphic Phantom, Alanine, Radiochromic film

\section{Highlights:}

- A multi-institutional end-to-end assessment of radiosurgery dosimetric delivery was performed for 33 plans in 30 participating centres using a variety of treatment platforms.

- The comparison has highlighted the dosimetric variation-consistency achievable between with different delivery platforms. The gantry-based linac group showed the greatest dose variation, compared to CK, and GK.

- The results showed clinically acceptable accuracy for all participants and illustrate the variation in the dosimetric accuracy achieved by various treatment platforms.

- The need for standardisation in intracranial stereotactic radiosurgery is highlighted.

\section{Abstract:}

\section{Background and Purpose:}

Assessment of dosimetric accuracy of radiosurgery on different treatment platforms. Material and Methods:

Thirty-three single fraction treatment plans were assessed at thirty centres using an anthropomorphic head phantom with target and brainstem structures. The target being a single irregular shaped target, $\sim 8 \mathrm{cc}, 10 \mathrm{~mm}$ from the brainstem. The phantom was "immobilised", scanned, planned and treated following the local protocols. EBT-XD films and alanine pellets were used to measure absolute dose, inside both the target and the brainstem, and compared with TPS predicted dose distributions.

Results:

PTV alanine measurements from gantry-based linacs showed the largest range ina median percentage difference to the TPS of 5.2\% (median $=0.65 \%$ ). Cyberknife (CK) had a range of $2.6 \%$ with the highest median difference of $(2.3 \%)$ in comparison to the other platforms. GammaKnife (GK) showed the smallest range and-median of $2.4 \%$ and $0.3 \%$ respectively. Similar trends were observed in the OAR with alanine measurements showing fanges and-median percentage differences ofs of $4.6 \%$ and $1.2 \%, 1.9 \%$ and $1.1 \%, 2.0 \%$ and $0.4 \%$, for gantry-based linacs, CK and GK respectively. All platforms showed comparable gamma passing rates between axial and sagittal films.

\section{Conclusions:}

This comparison has highlighted the dosimetric variation between measured and TPS calculated dose for each delivery platforms, with the gantry-based linac group showing the greatest dose variation, compared to CK, and GK.. However, $\underline{\text { Tthe }}$ 
Short title: Multi-institutional audit of dosimetric delivery in radiosurgery

43 results suggest that clinically acceptable agreement with the predicted dose 54 65 66

\section{7 \\ 1. Introduction}

49 Stereotactic radiosurgery (SRS), was first developed in the 1950s [1] and has since 50 evolved substantially. There are now several manufacturers that offer commercial 51 solutions for delivering SRS and such treatments may be delivered by eithera 52 Gamma Knife (GK) unit, a CyberKnife (CK) or a gantry-based linear accelerator 53 (linac) system with stereotactic capabilities.

55 All radiotherapy practices should be subjected to appropriate quality assurance 56 procedures, including regular quality control testing and independent external 57 dosimetry audit [2,3], to minimize potential errors in treatment delivery that can lead 58 to clinical complications [4]. This is especially the case for SRS where a very high 59 dose is delivered in only a single fraction, meaning an error cannot be mitigated in a 60 subsequent fraction.

62 The multiple platforms which can be used for SRS are very different in terms of their 63 technologies and techniques [5]. Furthermore, they have been ${ }_{2}$ and are being used $_{2}$ 64 very differently in terms of their dosimetric and clinical practices [6]. The

categorisation of the various systems into Gamma Knife (GK) units, CyberKnife (CK) units or gantry-based linear accelerator (linac) systems with stereotactic capabilities is justified according to their broadly similar dosimetric and clinical practices [6]. The latter category includes systems which use cones or tertiary microMLCs and also includes Ttomotherapy units, which use a fixed ring gantry and have no non-coplanar delivery capability.

This national study was undertaken to support an initiative in the UK to regulate the provision of cranial SRS services [7-9]. The participating centres were audited in an end-to-end test, incorporating local clinical procedures for immobilisation devices, CT-scanning, target contouring, treatment planning and treatment delivery. We evaluated the agreement between planned and delivered dose for each of the 
audited systems. It is acknowledged that the audited systems cover a wide range of delivery and ancillary systems, however such a study has the potential to benchmark what can be achieved with intracranial stereotactic surgery systems and therefore contribute data for use in setting tolerances for clinical trials and future external audits. Furthermore, the variations in clinical practice observed between these different platforms have been evaluated to determine-assess the need for standardisation.

\section{Materials and Methods}

A comprehensive end-to-end test was developed based on an anthropomorphic head phantom, STEEV (Stereotactic End-to-End Verification, CIRS, Norfolk, VI, USA). The phantom was adapted to contain a single irregularly shaped target ( 8cc), $10 \mathrm{~mm}$ anterior to the brainstem, for treatment by the audited centre following their local protocol for brain metastasis to be treated in a single fraction [10]. A Computed Tomography (CT) scan of the phantom was sent in advance to the participating centres in DICOM format for volume contouring and pre-planning. This was followed by a visit to the centre where the phantom was scanned following the local protocol (CT only), with eight dummy alanine pellets and two dummy pieces of EBT-XD Gafchromic film (Ashland ISP Advanced Materials, NJ, USA) placed inside the phantom to mimic the detector positions. These were subsequently replaced with the real detectors prior to phantom treatment irradiation. A graphical representation showing the target (PTV), brainstem (OAR) and detector positions is included in Figure 1. The two CT scans were co-registered, and the pre-plan transferred to the local scan and finalised. 
Short title: Multi-institutional audit of dosimetric delivery in radiosurgery

110 Thirty centres participated in the audit. In three centres two treatment platforms were assessed and hence thirty-three single fraction treatment plans were generated and delivered to the phantom. Table 1 provides details for each platform and plan that participated in the audit. Further details of the auditing protocol followed are given in Figure 2.

\subsection{Reference beam output measurements}

A calibrated PTW 31010 semiflex ionisation chamber $(0.125 \mathrm{cc})$, traceable to a graphite calorimeter primary standard at the National Physical Laboratory (NPL, Teddington, UK) was used in all centres to perform reference beam output measurements. The chamber was placed in an auditor provided water-equivalent plastic material, using the centre's reference conditions, to measure the output in the machine specific reference field [11]. All such auditor measurements were corrected for temperature and pressure. Temperature and pressure were measured using independent auditor equipment. The measurements were performed in machinespecific reference conditions and deviations from agreement were expressed relative to the TPS-calculated value for dose in a geometric water equivalent phantom.

The reference beam output measurements were used to apply corrections to the alanine measurements of the clinical plan, to compensate for any daily output variation.

\subsection{Alanine measurements}

Two sets of four alanine pellets, each a disc of $5 \mathrm{~mm}$ diameter and $2.5 \mathrm{~mm}$ thickness, were irradiated in each centre: one in the target and one in the brainstem. The phantom temperature before and after each measurement was recorded and used to apply a temperature correction factor. All pellets were returned to NPL and were processed within onet month of the audit visit, ensuring negligible fading [12]. The measured doses were compared to the mean dose calculated by the TPS for each pellet. Additionally, in order to account for positional uncertainties that can lead to large percentage differences between measured and predicted doses, mean doses for each pellet stack were compared with the TPS prediction for the complete stack.

141 The percentage difference between alanine and TPS for the OAR was normalised to 142 the 12 Gy dose level, being the nominal brainstem tolerance dose used by many 
centres. This enabled a dosimetric comparison whilst assessing the plan quality in terms of overdose to the OAR.

Uncertainty on the alanine readout was taken to be $1.4 \%(\mathrm{k}=2)[12]$. The deviations from agreement were expressed relative to the TPS-calculated value.

Alanine was considered as the primary detector for the audit measurements, as it has proven its efficacy in small fields and is provided by an established dosimetry service based at NPL [12].

\subsection{Film measurements}

The film response was calibrated in a conventional manner with ten EBT-XD film pieces exposed in the range of 0-40 Gy. The films were irradiated in a $10 \mathrm{~cm} \times 10$ $\mathrm{cm}$ field at $5 \mathrm{~cm}$ depth in water-equivalent plastic, with a nominal $6 \mathrm{MV}$ beam. The scanned pixel value as a function of dose was determined as the average pixel value in a $4 \times 4 \mathrm{~cm}$ region centred on the beam axis. Images were converted to dose maps using FilmQAPro® software (Ashland ISP Advanced Materials, NJ, USA) using the red-green-blue triple-channel dosimetry algorithm [13].

Two films (one sagittal and one axial) were placed inside the phantom and simultaneously irradiated at each centre. All films were returned to NPL and scanned at least 72 hours after exposure following an established film dosimetry protocol $[14,15]$ and good film dosimetry practice $[16,17]$ with the analysis performed on FilmQAPro. Film-dose linear scaling was applied using reference films at zero dose and $80 \%$ of the maximum anticipated dose from the treatment plan, which were scanned simultaneously with the test films. This approach mitigates the effects of post-exposure darkening and variations of the scanner response, and stabilizes the calibration (forced into agreement) at the reference dose levels [18]. The regions of interest used for the analysis were a $6 \times 5 \mathrm{~cm}$ rectangle for the axial films and a $7 \times 4$ $\mathrm{cm}$ rectangle for the sagittal films. The measured dose distributions were matched to TPS dose distributions by aligning the fiducial marker holes in the film with known coordinates in the TPS dose planes and then using the optimum shift algorithm, where small shifts were applied $\left(<1 \mathrm{~mm}\right.$ and $\left.<1^{\circ}\right)$ to optimise the gamma passing rate:-was achieved. 
174 Gamma passing rates [19] were collected for a range of criteria (global and local) using the red colour channel with triple-channel-correction and with film-dose linear scaling corrections applied. The criteria selected were based on their clinical significance for fadiosurgery and the typical dose gradients observedon the following:- dData for $3 \%$ dose difference and $2 \mathrm{~mm}$ distance $(3 \% / 2 \mathrm{~mm})$ were collected as this criterion was commonly used by the participating linac users and $5 \% / 1 \mathrm{~mm}$ as it this is more suitable for plans with steep gradients. Data for $2 \% / 2 \mathrm{~mm}$ was also analysed as recommended by AAPM TG 135 [20]. A minimum cut-off threshold of 2 Gy was applied to remove low dose areas of higher uncertainty from the analysis. This absolute value was used as an alternative to a relative value, due to the large variation of prescription doses and maximum doses delivered by the audit participants.

\subsection{Statistical analysis}

A Kruskali-Wallis test was performed to detect if there were any significant differences in median values between the three platforms, followed by the Bonferroni-type multiple comparison to establish the hierarchy/significant differences between different pairs.

\section{Results}

The-Rreference output measurements were performed independently by both the host centres and the auditors using their respective equipment and these are presented in Figure 3. All measurements from the auditors and the centres were within $\pm 2.4 \%$ of the dose calculated by the TPS, ranging from $-1.0 \%$ to $+2.4 \%$. The mean difference between auditor measurement and host centre measurement (audit/host) was $+0.5 \%$, with a maximum difference of $+1.2 \%$. The gantry based linac group had the largestspread in the output measurements of was $3.2 \%$, ranging from $-0.8 \%$ to $+2.4 \%$, with a median of $0.35 \%$. CKs had a smaller spread of $1.8 \%$, ranging from $1.0 \%$ to $+0.8 \%$, with a median of $0.85 \%$ and GKs had the smallesta of spread, $1.6 \%$, ranging from $-0.9 \%$ to $+0.7 \%$, with a median of $0.0 \%$. No statistical differences were seen between the platforms. 
The differences between the measured and calculated (measured/calculated) doses for the stack of 4 alanine pellets ranged from $-1.3 \%$ to $+4.0 \%$ for the PTV (Figure $4 \mathrm{~A}$ ). The gantry based linacs showed the largesta spread in percentage difference of $5.2 \%$ (from $-1.3 \%$ to $+3.9 \%$ ) with a median of $0.65 \%$. GKs showed the smallesta spread at of $2.4 \%$ (from $-0.8 \%$ to $+1.5 \%$ ), with the smallesta median percentage difference of $(+0.3 \%$ ). CK measurements had a spread of $2.6 \%$ (from $+1.4 \%$ to $+4 \%$ ), with the highesta median in comparison to the other platformsof $(+2.3 \%)$, which in comparison with the gantry based linac and GK groups were statistically higher with $p$ values of 0.045 and 0.039 respectively. Individual pellet measurements in the target showed differences of up to $14.1 \%, 4.2 \%$ and $5.9 \%$ (for GK, CK and linac respectively) when compared to the TPS-predicted mean dose in their individual contoured pellet structure.

Similar trends were observed in the comparison of OAR alanine pellet measurements to those observed in target alanine pellet measurements, ranging from $-1.1 \%$ to $+4.3 \%$ (Figure 4B). Gantry-based linac measurements showed the largesta spread at $4.6 \%$ ranging from $-1 \%$ to $+3.6 \%$ with a median of $+1.2 \%$. CK measurements ranged between $0.0 \%$ to $+1.9 \%$ with a median of $+1.1 \%$. GKs had a similarspread to CKsof $(2.0 \%)$ ranging from $-1.1 \%$ to $+0.9 \%$ but with a lower-and a- median of $+0.4 \%$. Here the only significance was in the gantry-based linac group being higher than the GK group with a $p$ value of 0.047 .

TPS-predicted doses for the alanine pellets in the OAR ranged from 0.3 Gy up to 7.5 Gy. Figure 4 shows the percentage difference between the mean dose measured by OAR alanine pellets with the TPS-predicted mean dose, normalised to 12.0 Gy.

The results for axial and sagittal films (example shown in Supplementary Figure 1) showed consistency in the gamma passing rates achieved. The gamma passing rates for $3 \% / 2 \mathrm{~mm}$ local gamma and $5 \% / 1 \mathrm{~mm}$ global gamma are shown in Figure 5.

Higher passing rates for all films were observed for global than for local gamma criteria. For the $3 \% / 2 \mathrm{~mm}$ local gamma criterion, all but two films showed passing 
rates above $75 \%$. The $\mathrm{CK}, \mathrm{GK}$ and gantry-based linac groups had median passing rates of $88.7,92.8$ and 85.5 respectively, showing no statistical differences between them. For the $5 \% / 1 \mathrm{~mm}$ global gamma criterion, all but 3 films showed passing rates above $90 \%$ (see Figure 5). Here the CK, GK and gantry-based linac groups had median passing rates of $99.3,98.4,98.3$ respectively, again showing no statistical differences between them. For the $2 \%$ / $2 \mathrm{~mm}$ global gamma criterion, all but 3 films showed passing rates above 90\% (see Supplementary Figure 2). Here the CK, GK and gantry-based linac groups had median passing rates of 99.3, 99.0, 96.6 respectively, with a p value of 0.028 between the GK and the gantry-based linac groups, all other comparisons being non-significant.

When the regions of interest used for the gamma analysis were reduced to smaller areas to include the target region only, passing rates improved substantiallyignificantly for all centres, showing very good agreement (>95\%) between TPS-predicted and delivered dose distributions. The majority of failed pixels for all films analysed were found to be outside the target, between the 2 Gy (threshold level) and the 12 Gy isodose line.

\section{Discussion}

The reference output measurements performed in the standard conditions for each platform at all centres were within $\pm 2.4 \%$ of the expected dose, well within the $\pm 3 \%$ recommendation of IPEM report 81 [21]. Moreover, the results seen in this study are consistent with the results reported by the NPL over 20 years of reference audits [22]. The differences from expected dose seen in the GK and CK groups were comparable, and much smaller than those seen in gantry-based linacs. This could be related to the smaller number of centres in these groups in comparison to the larger gantry-based linac group, but also due to-these platforms having more predictable output in reference conditions, due to having a simpler-more straight forward design. The ${ }^{60} \mathrm{Co}$ sources in the GK have a predictable decay, which is reflected in the good agreement seen in these measurements and the tighter tolerances of $1.0-1.5 \%$ deviation used by GK centres. The compact single energy linear accelerator of the CK may be the reason for the reduced fluctuations observed in output measurements, compared to 
multi-energy and multi-modality conventional gantry-based linacs which comprise the majority of units within this category. The systematic difference between the auditor and the centres $(+0.5 \%)$ may be partly explained by the instruments used. While the auditors used a $0.125 \mathrm{cc}$ ionisation chamber for all measurements, the centres used a range of different detectors, which in most cases had a larger sensitive volume. Therefore, a small degree of volume averaging can be attributed to the difference observed, especially since 15 out of 33 participating platforms used Flattening Filter Free (FFF) type beams. However, further investigation-measurements needs to be performed to fully explain this difference.

Overall, good agreement was observed between alanine and TPS, with three centres falling outside (greater than) two standard deviations of the mean (two centres in the target dose measurements and one in the OAR measurements). Although there were some statistical differences between the groups these had $p$ values which were only just less than 0.05 and were not consistent across the measurement methods and PTV/OAR, hence no strong statistical conclusions can be drawn about each platform. The gantry-based linac group was seen to have the largest spread in percentage differences compared to CK and GK, but with a relatively good overall agreement to with the TPS. The reasons behind this spread are most likely attributed to the diversity of techniques, platforms, beam energies, TPSs, calculation algorithms, clinician preferences and influences from the local radiotherapy practices. In comparison, the CK and GK groups had almost identical settings and practices within their subgroups. It is also possible that the variations in the commissioning methodologies used for gantry-based linacs, especially with regards to the dosimeters used for the measurement of small radiation fields, has an impact on the accuracy of deliverydose calculation. Although numerous studies have been conducted on appropriate detectors for small field applications [23-28], there has been a lack of international guidelines until the recently published IAEA TRS 483 [29]. It is expected that these new guidelines will improve the standardisation of commissioning methodologies.

CK measurements in the target, showed that the TPS with Raytracing calculation algorithm under-estimated the dose in all four centres visited. This finding is in agreement with another study utilising the same alanine service for target dose measurements in CK plans [30]. 
301

All GK centres used a TMR10 algorithm that does not account for density inhomogeneities and assumes water density within a CT-generated or depth-helmet measured skull contour. A recent study investigating the GK convolution algorithm (employs density corrections) in comparison to the TMR 10 , showed a $6 \%$ difference between the two where $1.5 \%$ of this was attributed to depth helmet measurements [31]. Our alanine measurements showed good agreement with the TPS and suggest that these sources of error do not contribute significantly to dosimetric inaccuracies. Further work investigating this convolution algorithm may be required to evaluate its accuracy before it is used clinically.

Alanine measurements in the OAR were performed along a steep dose gradient where any positional uncertainties may be expressed as large dose differences. Doses to the OAR pellets ranged by up to an order of magnitude, caused by individual planning priorities and protocols used by participating centres. As some centres delivered very low doses to this region the reported doses were normalised to 12 Gy (a nominal brainstem tolerance dose value used by many centres) in order to provide a useful measure of relative accuracy to the centres with different approaches. There was also a higher uncertainty in the lower dose measurements due to lower signal to noise ratios in the alanine readout.

The gamma passing rates showed clinically acceptable agreement between the filmmeasured dose and the treatment planning system calculated dose distributions for both sagittal and axial films [32]. All treatment modalities showed comparable variations in passing rates between the centres assessed and the passing rates alone do not suggest significant differences between the different platforms. Other studies have suggested that gamma index analysis is non-ideal for direct comparison in multiinstitution assessments due to inherent differences in the dose distributions, particularly in dose gradient and maximum doses [32-34]. Local Gamma criteria may favour linac centres in which the dose gradient could be less steep than GK and CK, and Global Gamma criteria may favour GK centres in which the maximum (normalisation) dose is higher and the dose gradients are steeper. Whilst the methodology for film analysis employed in this study was designed to diminish sensitivities to different dose distributions it is impossible to achieve this with gamma index analysis. Gamma passing rates are also sensitive to the position of the film 
relative to the dose plan, the position of the region of interest used for the analysis and the 2 Gy threshold levels applied. An analysis method that is less sensitive to these dose distribution differences could be preferable, enabling a more reliable direct comparison between competing plans. Future studies of this nature must develop novel methodologies to enable more meaningful and clinically relevant comparisons, such as 3D dosimetry and improvements in the analysis of dose distributions moving towards a DVH-based assessment. The use of dose-plane-histograms (analogous to dose-volume histograms (DVH) in a single plane) may provide a more clinically relevant analysis [35,36]. Despite its pitfalls, the gamma analysis method used, enabled quantification of the dose shaping abilities of all SRS platforms active in the UK. The results showed clinically acceptable dosimetric performance by all platforms, although noticeable dosimetric differences were apparent outside the target volume, which are unlikely to be clinically relevant. These dosimetric inaccuracies, seen in most centres, are related to the TPS's limitations in simulating out of field doses and typically resulted in underestimation of doses to the OAR, as found by other studies [37]. Another study conducting film-based end-to-end tests in CK plans recorded higher gamma passing rates (>90\%) for the criterion of $2 \%-2 \mathrm{~mm}$ local gamma [38], compared to the median passing rates seen in this study of 76.8, 81.0 and $75.1 \%$ for CK, GK and gantry-based linacs respectively. The differences seen are explained by the higher $50 \%$ dose threshold used in that study that excludes the low doses included in our film analysis.

While other multi-platform assessments have been conducted on stereotactic applications [30,39,40] only a few have been performed specifically for SRS [41]. This study is novel in the diversity of treatment platforms included and the advanced dosimetry methods employed. During-In this study, thirty-three treatment plans with differing planning approaches were generated, all for the same realistic patient scenario of a single metastatic lesion located anterior to the brainstem. The approaches adopted by the participants to treat the presented indication differed in many aspects (see Table 1). Some of these differences, with respect to the equipment, software and delivery techniques used, were previously identified [6]. Aside from these planning differences, some subtle differences were observed in the accuracy of the measured dose distributions. However, the most influential and clinically relevant 
Short title: Multi-institutional audit of dosimetric delivery in radiosurgery

365 variation observed in the protocols assessed, was found in prescription practices (Table 1), highlighting the potential need for standardisation.

\section{This study assessed the dosimetric accuracy achieved using the thirty-three} participating platforms. Although a statistical analysis was performed, there were-small numbers in each group and a more robust analysis can be performed withmeans a larger population would be needed for a more robust analysis. However, this was a national study and a larger study can-could only be realised at a multi-national level. A Such a larger study may also reveal more subtle differences in the individual approaches followed in each centre. Another limitation of this work is the lack of incorporation of MRI in the end-to-end assessment, which is an integral step in intracranial SRS. As mentioned previously, there are also limitations with the use of gamma analysis in this setting. Future studies of this nature must develop novel methodologies to enable more meaningful and clinically relevant comparisons, such as 3D dosimetry and improvements in the analysis of dose distributions moving towards a DVH based assessment.

With the recent rise of gantry based linac SRS [6] it is essential to incorporate all SRS platforms in dosimetric studies and clinical trials, in order to reach consensus in several matters. SRS treatments have been traditionally split between neurosurgeryled single session SRS and multisession oncology-led practices. However, the promising outcomes seen in hypofractionated SRS [42] and staged-SRS [43] has brought the two faculties closer. In the future stereotactic trials should consider the diversity of platforms that may be used, in particular for issues such as prescription dose, prescription isodose, delivery capability, and how dosimetric differences may be assessed [44].

Currently, the assessment of dosimetric deliveries in a multi-platform, multi-centre SRS setting with individual planning priorities remains a challenge. However, independent dosimetric assessments as presented in this study are important interventions which have a crucial role in ensuring accurate dose delivery to patients. Moreover, when data from multiple centres is pooled together, it enables participants to benchmark their services against the rest of their community, assess their safety, evaluate their practices and consider improvements to their service. As stated the 
1

2

3

basis of this audit was to support an initiative in the UK to regulate the provision of cranial SRS services. Consistent dosimetry has been recognised as essential in the evaluation of outcomes from those centres commissioned to provide such clinical services. Overall, there was a smaller spread of data seen in the CK and GK groups, however each had some statistically significant differences with the other platforms.

This suggests that Ffuture multicentre SRS studies may benefit from some standardisation and consensus of practice.

\section{Acknowledgments}

Funding for PhD studies and partial travel costs - Engineering \& Physical Sciences Research Council (EPSRCEP/J500094), Technical support (llias Billas, Michael Homer, Ana Subiel, Simon Duane, Sebastian Galer), Alanine analysis (Gavin Cox, David Crossley), Audit support (Elizabeth Miles, Rada Zotova), Pilot audit support (Martyn Gilmore, Phillip Cooper, Kelvin Hiscoke).

\section{References}

[1] Leksell L. The stereotaxic method and radiosurgery of the brain. Acta Chir Scand 1951;102:316-9.

[2] Clark $\mathrm{CH}$, Jornet $\mathrm{N}$, Muren LP. The role of dosimetry audit in achieving high quality radiotherapy 2018. https://doi.org/10.1016/j.phro.2018.03.009.

[3] Clark CH, Ga Aird E, Bolton S, Miles EA, Nisbet A, Snaith JA, et al. Radiotherapy dosimetry audit: Three decades of improving standards and accuracy in UK clinical practice and trials. Br J Radiol 2015;88. https://doi.org/10.1259/bjr.20150251.

[4] Briggs G, Ebdon-Jackson S, Erridge SC, Graveling M, Hood S, McKenzie A, et al. Towards Safer Radiotherapy. R Coll Radiol Soc Coll Radiogr Inst Phys Eng Med Natl Patient Saf Agency Br Inst Radiol 2008:85. https://doi.org/978-1905034-25-3. 
[5] Kocher M, Wittig A, Piroth MD, Treuer H, Seegenschmiedt $\mathrm{H}$, Ruge M, et al. Stereotactic radiosurgery for treatment of brain metastases: A report of the DEGRO Working Group on Stereotactic Radiotherapy. Strahlentherapie Und Onkol 2014;190:521-32. https://doi.org/10.1007/s00066-014-0648-7.

[6] Dimitriadis A, Kirkby KJ, Nisbet A, Clark CH. Current Status of Cranial Stereotactic Radiosurgery in the UK. Br J Radiol 2016;89:20150452. https://doi.org/10.1259/bjr.20150452.

[7] NHS England. Stereotactic Radiosurgery/ Stereotactic Radiotherapy Needs Assessment and Service Review Consultation Report. 2015.

[8] NHS England. Service Specifications: Stereotactic radiosurgery and stereotactic radiotherapy (Intracranial) (All ages). London: 2016.

[9] Eaton DJ, Lee J, Paddick I. Stereotactic radiosurgery for multiple brain metastases: Results of multicenter benchmark planning studies. Pract Radiat Oncol 2018;0. https://doi.org/10.1016/j.prro.2017.12.011.

[10] Dimitriadis A, Palmer AL, Thomas RAS, Nisbet A, Clark CH. Adaptation and validation of a commercial head phantom for cranial radiosurgery dosimetry end-to-end audit. Br J Radiol 2017;90:20170053. https://doi.org/10.1259/bjr.20170053.

[11] Alfonso R, Andreo P, Capote R, Huq MS, Kilby W, Kjäll P, et al. A new formalism for reference dosimetry of small and nonstandard fields. Med Phys 2008;35:5179-86. https://doi.org/10.1118/1.3005481.

[12] Sharpe PHG, Sephton JP. Therapy level alanine dosimetry at the NPL. Proc. 216th PTB Semin. Alanine Dosim. Clin. Appl. PTB-Dos-51, PTB, Braunschweig, 2006.

[13] Micke A, Lewis DF, Yu X. Multichannel film dosimetry with nonuniformity correction. Med Phys 2011;38:2523-34. https://doi.org/10.1118/1.3611636.

[14] Palmer AL, Dimitriadis A, Nisbet A, Clark CH. Evaluation of Gafchromic EBTXD film, with comparison to EBT3 film, and application in high dose radiotherapy verification. Phys Med Biol 2015;60:8741-52. https://doi.org/10.1088/0031-9155/60/22/8741.

[15] Dimitriadis A. Assessing the dosimetric and geometric accuracy of stereotactic radiosurgery. University of Surrey, 2017.

[16] Niroomand-Rad A, Blackwell CR, Coursey BM, Gall KP, Galvin JM, McLaughlin WL, et al. Radiochromic film dosimetry: Recommendations of AAPM Radiation Therapy Committee Task Group 5. Med Phys 1998;25:2093115. https://doi.org/10.1118/1.598869.

[17] Palmer A, Bradley D, Nisbet A. Evaluation and mitigation of potential errors in radiochromic film dosimetry due to film curvature at scanning. J Appl Clin Med 2015;16:425-31.

[18] Lewis D, Micke A, Yu X, Chan MF. An efficient protocol for radiochromic film dosimetry combining calibration and measurement in a single scan. Med Phys 2012;39:6339-50. https://doi.org/10.1118/1.4754797.

[19] Low DA, Harms WB, Mutic S, Purdy JA. A technique for the quantitative evaluation of dose distributions. Med Phys 1998;25:656-61. https://doi.org/10.1118/1.598248.

[20] Dieterich S, Cavedon C, Chuang CF, Cohen AB, Garrett JA, Lee CL, et al. Report of AAPM TG 135: Quality assurance for robotic radiosurgery. Med Phys 2011;38:2914-36. https://doi.org/10.1118/1.3579139.

[21] Lillicrap SC. Physics Aspects of Quality Control in Radiotherapy (Report No. 81). Phys Med Biol 2000;45:815-815. https://doi.org/10.1088/0031- 
Short title: Multi-institutional audit of dosimetric delivery in radiosurgery

9155/45/3/501.

[22] Thomas RAS, Bolt MA, Bass G, Nutbrown R, Chen T, Nisbet A, et al. Radiotherapy reference dose audit in the United Kingdom by the National Physical Laboratory: 20 years of consistency and improvements. Phys Imaging Radiat Oncol 2017;3:21-7. https://doi.org/10.1016/j.phro.2017.07.005.

[23] Silvestre I, Dimitriadis A, Subiel A. Relative output factors for TPS beam data acquisition with emphasis on small fields. Comparison of multiple detectors and multiple approaches. Rev Latinoam Fis Medica - ISSN 2413-9904 2017;3.

[24] Dimitriadis A, Patallo Silvestre I, Billas I, Duane S, Nisbet A, Clark CH, et al. Characterisation of a plastic scintillation detector to be used in a multicentre stereotactic radiosurgery audit. Radiat Phys Chem 2017:0-1. https://doi.org/10.1016/j.radphyschem.2017.02.023.

[25] Bassinet C, Huet C, Derreumaux S, Brunet G, Chéa M, Baumann M, et al. Small fields output factors measurements and correction factors determination for several detectors for a CyberKnife $\AA$ and linear accelerators equipped with microMLC and circular cones. Med Phys 2013;40:071725. https://doi.org/10.1118/1.4811139.

[26] Kamio Y, Bouchard H. Correction-less dosimetry of nonstandard photon fields: A new criterion to determine the usability of radiation detectors. Phys Med Biol 2014;59:4973-5002. https://doi.org/10.1088/0031-9155/59/17/4973.

[27] Russo S, Masi L, Francescon P, Frassanito MC, Fumagalli ML, Marinelli M, et al. Multicenter evaluation of a synthetic single-crystal diamond detector for CyberKnife small field size output factors. Phys Medica 2016. https://doi.org/10.1016/j.ejmp.2016.03.005.

[28] Pimpinella M, Ciancaglioni I, Consorti R, Venanzio C Di, Guerra a S, Petrucci a, et al. A synthetic diamond detector as transfer dosimeter for $\mathrm{D} w$ measurements in photon beams with small field sizes. Metrologia 2012;49:S207-10. https://doi.org/10.1088/0026-1394/49/5/S207.

[29] Palmans H, Andreo P, Huq S, Seuntjens J. Dosimetry of small static fields used in external beam radiotherapy: An IAEA-AAPM International Code of Practice for reference and relative dose determination. Technical Report Series No. 483. laea Trs483 2017.

[30] Distefano G, Lee J, Jafari S, Gouldstone C, Baker C, Mayles H, et al. A national dosimetry audit for stereotactic ablative radiotherapy in lung. Radiother Oncol:406-10. https://doi.org/10.1016/j.radonc.2016.12.016.

[31] Rojas-villabona A, Kitchen N, Paddick I. Investigation of dosimetric differences between the TMR 10 and convolution algorithm for Gamma Knife stereotactic radiosurgery. J Appl Clin Med Phys 2016;17:1-13.

[32] Miften M, Olch A, Mihailidis D, Moran J, Pawlicki T, Molineu A, et al. Tolerance limits and methodologies for IMRT measurement-based verification QA:

Recommendations of AAPM Task Group No. 218. Med Phys 2018;45:e53-83. https://doi.org/10.1002/mp.12810.

[33] Ezzell GA, Burmeister JW, Dogan N, LoSasso TJ, Mechalakos JG, Mihailidis $\mathrm{D}$, et al. IMRT commissioning: Multiple institution planning and dosimetry comparisons, a report from AAPM Task Group 119. Med Phys 2009;36:535973. https://doi.org/10.1118/1.3238104.

[34] Hussein M, Clark $\mathrm{CHH}$, Nisbet A. Challenges in calculation of the gamma index in radiotherapy - Towards good practice. Phys Medica 2017;36:1-11. https://doi.org/10.1016/j.ejmp.2017.03.001.

[35] Palmer AL, Nash D, Kearton JR, Jafari SM, Muscat S. A multicentre "end to 
end" dosimetry audit of motion management (4DCT-defined motion envelope) in radiotherapy. Radiother Oncol 2017;125:453-8. https://doi.org/10.1016/j.radonc.2017.09.033.

[36] Sothmann T, Blanck O, Poels K, Werner R, Gauer T. Real time tracking in liver SBRT: Comparison of CyberKnife and Vero by planning structure-based $\gamma^{-}$ evaluation and dose-area-histograms. Phys Med Biol 2016;61:1677-91. https://doi.org/10.1088/0031-9155/61/4/1677.

[37] Kry SF, Bednarz B, Howell RM, Dauer L, Followill D, Klein E, et al. AAPM TG 158: Measurement and calculation of doses outside the treated volume from external-beam radiation therapy. Med Phys 2017;44:e391-429. https://doi.org/10.1002/mp.12462.

[38] Blanck O, Masi L, Damme M-C, Hildebrandt G, Dunst J, Siebert F-A, et al. Film-based delivery quality assurance for robotic radiosurgery: Commissioning and validation. Phys Medica 2015;31:476-83. https://doi.org/10.1016/j.ejmp.2015.05.001.

[39] Lambrecht M, Melidis C, Sonke J-J, Adebahr S, Boellaard R, Verheij M, et al. Lungtech, a phase II EORTC trial of SBRT for centrally located lung tumours a clinical physics perspective. Radiat Oncol 2016;11:7. https://doi.org/10.1186/s13014-015-0567-5.

[40] Kron T, Chesson B, Hardcastle N, Crain M, Clements N, Burns M, et al. Credentialing of radiotherapy centres in Australasia for TROG 09.02 (Chisel), a Phase III clinical trial on stereotactic ablative body radiotherapy of early stage lung cancer. Br J Radiol 2018:20170737. https://doi.org/10.1259/bjr.20170737.

[41] Seravalli E, Van Haaren PMA, Van Der Toorn PP, Hurkmans CW. Intercranial stereotactic RT A comprehensive evaluation of treatment accuracy, including end-to-end tests and clinical data, applied to intracranial stereotactic radiotherapy. Radiother Oncol 2015;116:131-8. https://doi.org/10.1016/j.radonc.2015.06.004.

[42] Ishihara T, Yamada K, Harada A, Isogai K, Tonosaki Y, Demizu Y, et al. Hypofraktionierte stereotaktische Strahlentherapie bei Hirnmetastasen eines Lungenkarzinoms: Evaluierung von Indikationen und Prädiktoren der lokalen Kontrolle. Strahlentherapie Und Onkol 2016;192:386-93. https://doi.org/10.1007/s00066-016-0963-2.

[43] Higuchi Y, Serizawa T, Nagano O, Matsuda S, Ono J, Sato M, et al. Threestaged stereotactic radiotherapy without whole brain irradiation for large metastatic brain tumors. Int J Radiat Oncol Biol Phys 2009:74:1543-8. https://doi.org/10.1016/j.ijrobp.2008.10.035.

[44] Wilke L, Andratschke N, Blanck O, Brunner TB, Combs SE, Grosu AL, et al. ICRU report 91 on prescribing, recording, and reporting of stereotactic treatments with small photon beams: Statement from the DEGRO/DGMP working group stereotactic radiotherapy and radiosurgery. Strahlentherapie Und Onkol 2019;195:193-8. https://doi.org/10.1007/s00066-018-1416-*. 


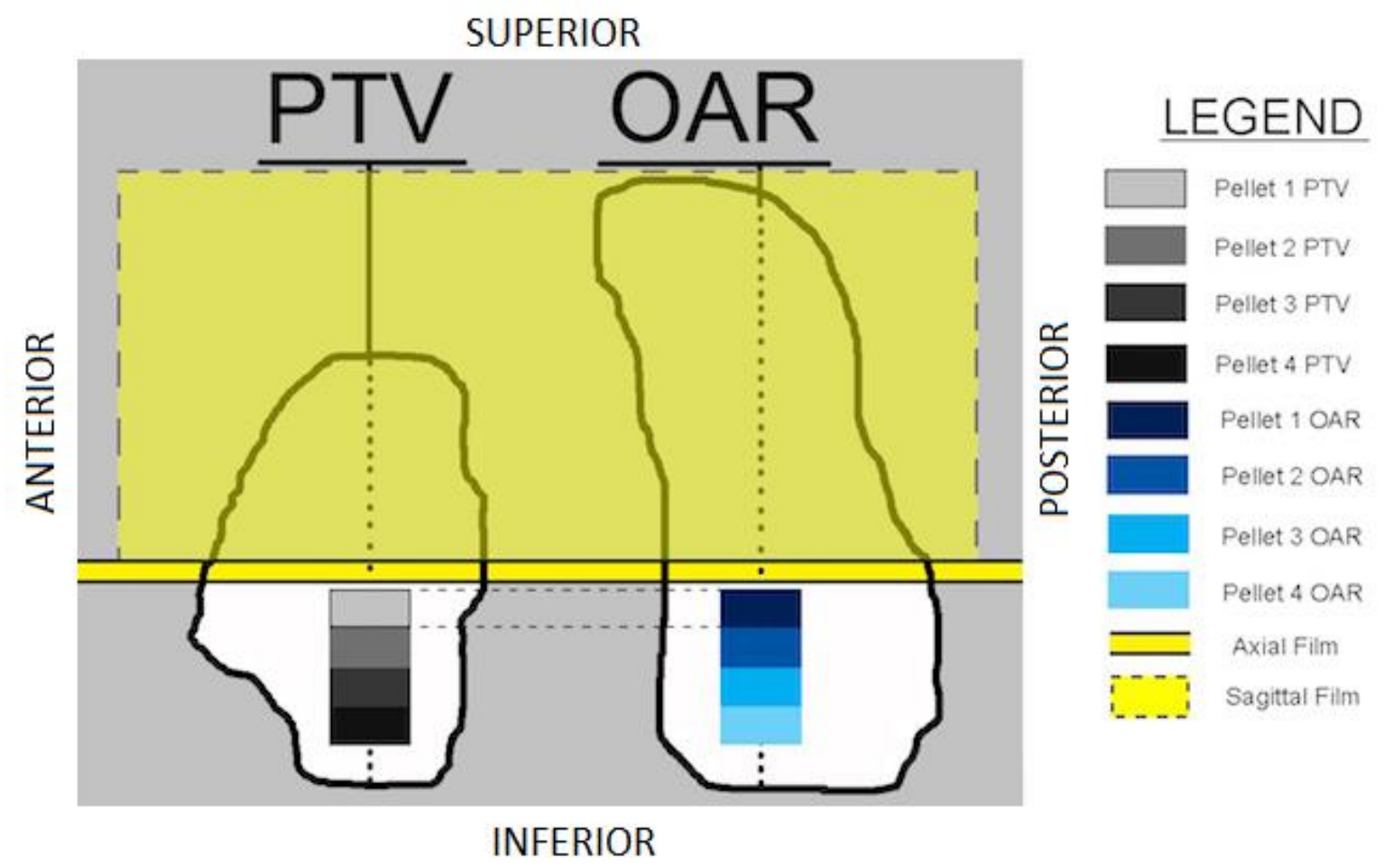

Figure 1: Schematic representation of a sagittal view through the centre of the phantom, showing the positions of the detectors in relation to the target and brainstem structures. 
Short title: Multi-institutional audit of dosimetric delivery in radiosurgery

1

2

3

4

5

6

7

8

9

10

11

12

13

14

15

16

17

18

19

20

21

22

23

24

25

26

27

28

29

30

31

32

33

34

35

36

37

38

39

40

41

\begin{tabular}{|c|c|c|c|c|c|c|c|c|c|c|}
\hline $\begin{array}{l}\text { Centre } \\
\text { Platfor } \\
\text { m No. }\end{array}$ & Platform & Energy & Technique & $\begin{array}{l}\text { Coplanar } \\
\text { / Non- } \\
\text { coplanar }\end{array}$ & TPS & $\begin{array}{c}\text { Dose } \\
\text { Calculation } \\
\text { Algorithm }\end{array}$ & $\begin{array}{c}\text { Density } \\
\text { Heterog. } \\
\text { Correction }\end{array}$ & $\begin{array}{c}\text { Peripheral } \\
\text { Prescr. } \\
\text { Dose (Gy) }\end{array}$ & $\begin{array}{l}\text { Max } \\
\text { Dose } \\
\text { (Gy) }\end{array}$ & $\begin{array}{c}\text { Pr Iso. } \\
\text { Relative to } \\
\text { Dmax (\%) }\end{array}$ \\
\hline 1 & $\begin{array}{c}\text { VRN } \\
\text { TrueBeam } \\
\text { STx } \\
\end{array}$ & 10MV FFF & 4DCA & $\mathrm{NC}$ & BL iPlan & $\begin{array}{l}\text { Pencil } \\
\text { Beam }\end{array}$ & Yes & 21.0 & 26.4 & $80 \%$ \\
\hline 2 & BL Novalis Tx & $6 \mathrm{MV}$ & $9 \mathrm{FF}$ & $\mathrm{NC}$ & BL iPlan & $\begin{array}{l}\text { Pencil } \\
\text { Beam }\end{array}$ & Yes & 17.5 & 22.7 & $77 \%$ \\
\hline 3 & VRN 2100x & $6 \mathrm{MV}$ & $4 \mathrm{CCA}$ & $\mathrm{NC}$ & BL iPlan & $\begin{array}{c}\text { Circular } \\
\text { Cone }\end{array}$ & Yes & 18.0 & 23.6 & $76 \%$ \\
\hline 4 & ELK Synergy & $6 \mathrm{MV}$ & 5VMAT & $\mathrm{NC}$ & Pinnacle & Convolution & Yes & 18.0 & 22.9 & $79 \%$ \\
\hline 5 & $\begin{array}{c}\text { ACC } \\
\text { Tomotherapy }\end{array}$ & $6 \mathrm{MV}$ FFF & $\begin{array}{l}\text { Tomo } \\
\text { Therapy }\end{array}$ & C & $\begin{array}{l}\text { Tomo } \\
\text { Therapy }\end{array}$ & $\begin{array}{l}\text { Non-Voxel } \\
\text { Broad } \\
\text { Beam }\end{array}$ & Yes & 18.0 & 20.7 & $87 \%$ \\
\hline 6 & BL Novalis Tx & 6MV SRS & $8 \mathrm{FF}$ & $\mathrm{NC}$ & Pinnacle & Convolution & Yes & 18.0 & 22.4 & $80 \%$ \\
\hline 7 & ELK Agility & $6 \mathrm{MV}$ & $3 \mathrm{DCA}$ & $\mathrm{NC}$ & Monaco & MC Photon & Yes & 18.0 & 19.9 & $90 \%$ \\
\hline 8 & BL Novalis Tx & $6 \mathrm{MV}$ SRS & 4DCA & $\mathrm{NC}$ & BL iPlan & $\begin{array}{c}\text { Pencil } \\
\text { Beam }\end{array}$ & Yes & 18.0 & 23.1 & $78 \%$ \\
\hline 9 & $\begin{array}{c}\text { VRN } \\
\text { TrueBeam }\end{array}$ & $6 \mathrm{MV}$ & 4DCA & $\mathrm{NC}$ & BL iPlan & $\begin{array}{l}\text { Pencil } \\
\text { Beam }\end{array}$ & Yes & 18.0 & 22.8 & $79 \%$ \\
\hline 10 & $\begin{array}{c}\text { ELK } \\
\text { VersaHD }\end{array}$ & $6 \mathrm{MV}$ & 5VMAT & $\mathrm{NC}$ & Monaco & MC Photon & Yes & 21.0 & 26.3 & $80 \%$ \\
\hline 11 & $\begin{array}{c}\text { VRN } \\
\text { TrueBeam }\end{array}$ & 10MV FFF & 2VMAT & C & Eclipse & AAA & Yes & 20.0 & 23.3 & $86 \%$ \\
\hline 12 & BL Novalis Tx & $6 \mathrm{MV}$ SRS & 4DCA & $\mathrm{NC}$ & BL iPlan & $\begin{array}{l}\text { Pencil } \\
\text { Beam }\end{array}$ & Yes & 18.0 & 23.2 & $78 \%$ \\
\hline 13 & $\begin{array}{c}\text { VRN } \\
\text { TrueBeam }\end{array}$ & $6 \mathrm{MV}$ FFF & 1VMAT & C & Eclipse & AAA & Yes & 16.0 & 19.9 & $80 \%$ \\
\hline 14 & $\begin{array}{c}\text { VRN } \\
\text { TrueBeam }\end{array}$ & 10MV FFF & 1VMAT & C & Eclipse & AAA & Yes & 16.0 & 20.3 & $79 \%$ \\
\hline 15 & $\begin{array}{c}\text { ELK } \\
\text { BeamMod }\end{array}$ & $6 \mathrm{MV}$ & $8 \mathrm{FF}$ & NC & Pinnacle & Convolution & Yes & 21.0 & 23.5 & $89 \%$ \\
\hline 16 & $\begin{array}{c}\text { VRN } \\
\text { TrueBeam } \\
\text { STx }\end{array}$ & $6 \mathrm{MV}$ & $5 \mathrm{DCA}$ & $\mathrm{NC}$ & BL iPlan & $\begin{array}{l}\text { Pencil } \\
\text { Beam }\end{array}$ & Yes & 18.0 & 22.7 & $79 \%$ \\
\hline 17 & $\begin{array}{c}\text { ELK } \\
\text { VersaHD }\end{array}$ & $6 \mathrm{MV}$ FFF & 3VMAT & $\mathrm{NC}$ & Monaco & MC Photon & Yes & 18.0 & 34.8 & $52 \%$ \\
\hline 18 & $\begin{array}{c}\text { VRN } \\
\text { TrueBeam }\end{array}$ & 10MV FFF & 4VMAT & $\mathrm{NC}$ & Eclipse & Acuros & Yes & 20.0 & 30.0 & $67 \%$ \\
\hline 19 & $\begin{array}{c}\text { ELK } \\
\text { BeamMod }\end{array}$ & $6 \mathrm{MV}$ & 7DCA & $\mathrm{NC}$ & Pinnacle & Convolution & Yes & 18.0 & 24.1 & $75 \%$ \\
\hline 20 & $\begin{array}{c}\text { VRN } \\
\text { TrueBeam } \\
\text { STx }\end{array}$ & $6 \mathrm{MV}$ & $5 \mathrm{DCA}$ & $\mathrm{NC}$ & BL iPlan & $\begin{array}{l}\text { Pencil } \\
\text { Beam }\end{array}$ & Yes & 21.0 & 27.1 & $77 \%$ \\
\hline 21 & VRN ix2100 & $6 \mathrm{MV}$ & 7VMAT & $\mathrm{NC}$ & Eclipse & AAA & Yes & 21.0 & 30.5 & $69 \%$ \\
\hline 22 & $\begin{array}{c}\text { VRN } \\
\text { TrueBeam } \\
\text { STx }\end{array}$ & $6 \mathrm{MV}$ FFF & $5 \mathrm{DCA}$ & NC & BL iPlan & $\begin{array}{l}\text { Pencil } \\
\text { Beam }\end{array}$ & Yes & 18.0 & 22.2 & $81 \%$ \\
\hline 23 & $\begin{array}{c}\text { ELK GK } \\
\text { Perfexion }\end{array}$ & ${ }^{60} \mathrm{Co}$ & 17 shots & NC & $\begin{array}{c}\text { Gamma } \\
\text { Plan }\end{array}$ & TMR10 & No & 18.0 & 40.9 & $44 \%$ \\
\hline 24 & $\begin{array}{c}\text { ELK GK } \\
\text { Perfexion }\end{array}$ & ${ }^{60} \mathrm{Co}$ & 19 shots & $\mathrm{NC}$ & $\begin{array}{c}\text { Gamma } \\
\text { Plan }\end{array}$ & TMR10 & No & 20.0 & 40.0 & $50 \%$ \\
\hline 25 & ELK GK Icon & ${ }^{60} \mathrm{Co}$ & 20 shots & NC & $\begin{array}{c}\text { Gamma } \\
\text { Plan }\end{array}$ & TMR10 & No & 18.0 & 36.7 & $49 \%$ \\
\hline 26 & ELK GK Icon & ${ }^{60} \mathrm{Co}$ & 22 shots & $\mathrm{NC}$ & $\begin{array}{c}\text { Gamma } \\
\text { Plan }\end{array}$ & TMR10 & No & 18.0 & 36.0 & $50 \%$ \\
\hline 27 & $\begin{array}{c}\text { ELK GK } \\
\text { Perfexion }\end{array}$ & ${ }^{60} \mathrm{Co}$ & 11 shots & NC & $\begin{array}{c}\text { Gamma } \\
\text { Plan }\end{array}$ & TMR10 & No & 18.0 & 39.1 & $46 \%$ \\
\hline 28 & $\begin{array}{l}\text { ELK GK } \\
\text { Perfexion }\end{array}$ & ${ }^{60} \mathrm{Co}$ & 22 shots & $\mathrm{NC}$ & $\begin{array}{c}\text { Gamma } \\
\text { Plan }\end{array}$ & TMR10 & No & 18.0 & 40.9 & $44 \%$ \\
\hline 29 & $\begin{array}{c}\text { ELK GK } \\
\text { Perfexion }\end{array}$ & ${ }^{60} \mathrm{Co}$ & 22 shots & $\mathrm{NC}$ & $\begin{array}{c}\text { Gamma } \\
\text { Plan }\end{array}$ & TMR10 & No & 18.0 & 41.4 & $43 \%$ \\
\hline 30 & ACC CK VSI & $6 \mathrm{MV}$ FFF & 138 beams & $\mathrm{NC}$ & $\begin{array}{l}\text { Multi } \\
\text { Plan }\end{array}$ & Ray Tracing & Yes & 21.0 & 32.3 & $65 \%$ \\
\hline 31 & ACC CK VSI & $6 \mathrm{MV}$ FFF & 123 beams & $\mathrm{NC}$ & $\begin{array}{l}\text { Multi } \\
\text { Plan }\end{array}$ & Ray Tracing & Yes & 18.0 & 25.7 & $70 \%$ \\
\hline 32 & ACC CK VSI & 6MV FFF & 139 beams & $\mathrm{NC}$ & $\begin{array}{l}\text { Multi } \\
\text { Plan }\end{array}$ & Ray Tracing & Yes & 18.0 & 34.0 & $53 \%$ \\
\hline 33 & ACC CK VSI & $6 \mathrm{MV}$ FFF & 109 beams & NC & $\begin{array}{l}\text { Multi } \\
\text { Plan }\end{array}$ & Ray Tracing & Yes & 20.0 & 30.8 & $65 \%$ \\
\hline
\end{tabular}

608 Table 1: Summary of equipment, techniques and prescription practices of the audit participants. The 609 centres are grouped by platform and in random order, different to the order shown in the results, to 610 avoid identification of individual centres. On-board imaging for positioning the phantom was used by all 611 participants except centres 3, 23, 24, 27, 28 and 29. (VRN=Varian, BL=Brainlab, ELK=Elekta,

612 ACC=Accuray, GK=Gamma Knife, CK=Cyberknife (with cones), DCA=Dynamic Conformal Arcs, 613 FF=Fixed Fields, $C C A=$ Circular Collimator Arcs, VMAT=Volumetric Modulated Arc Therapy) 


\section{Pre- assessment}

1. A high resolution DICOM image set of the phantom was send to the host centre with instructions for contouring the target and the brainstem.

2. The host centre outlined the structures and reported back to auditors.

3. After approval from the auditors, the host centre practised on the planning scenario available and prepared a plan prior to the day of the audit.

\section{On day of assessment}

1. The phantom was presented to the host centre with dummy film and alanine pellets in place.

2. The immobilisation device (frame/mask) was applied to the phantom followed by a CTscan in stereotactic conditions following the local SRS protocol.

3. The acquired scan was fused/co-registered with the previously sent image set and the alanine pellets were delineated.

4. The plan was recalculated on the CT scan acquired on the day and exported to the treatment platform for delivery.

5. Output measurements in reference conditions were performed by both the auditor and auditee.

6. The dummy film and alanine pellets were replaced with real film and alanine pellets.

7. The audit phantom was accurately positioned on the treatment platform, with/without the use of on-board imaging, and the plan was delivered.

\section{Post- assessment}

1. The participating centre provided the auditors with RTDOSE DICOM files and dose-volume histograms from the delivered treatment plan for comparison to the measured alanine and films.

2. Alanine pellets and films were returned to the responsible laboratories for analysis

3. Alanine and film measured doses were compared to the TPS predicted doses.

4. The results were checked by a second person and a provisional report with the results was prepared and sent to the participating centre.

Figure 2: Flowchart diagram showing the main steps of the procedure followed in performing 
Short title: Multi-institutional audit of dosimetric delivery in radiosurgery

1
625 Output Measurements in Machine-Specific Reference beam

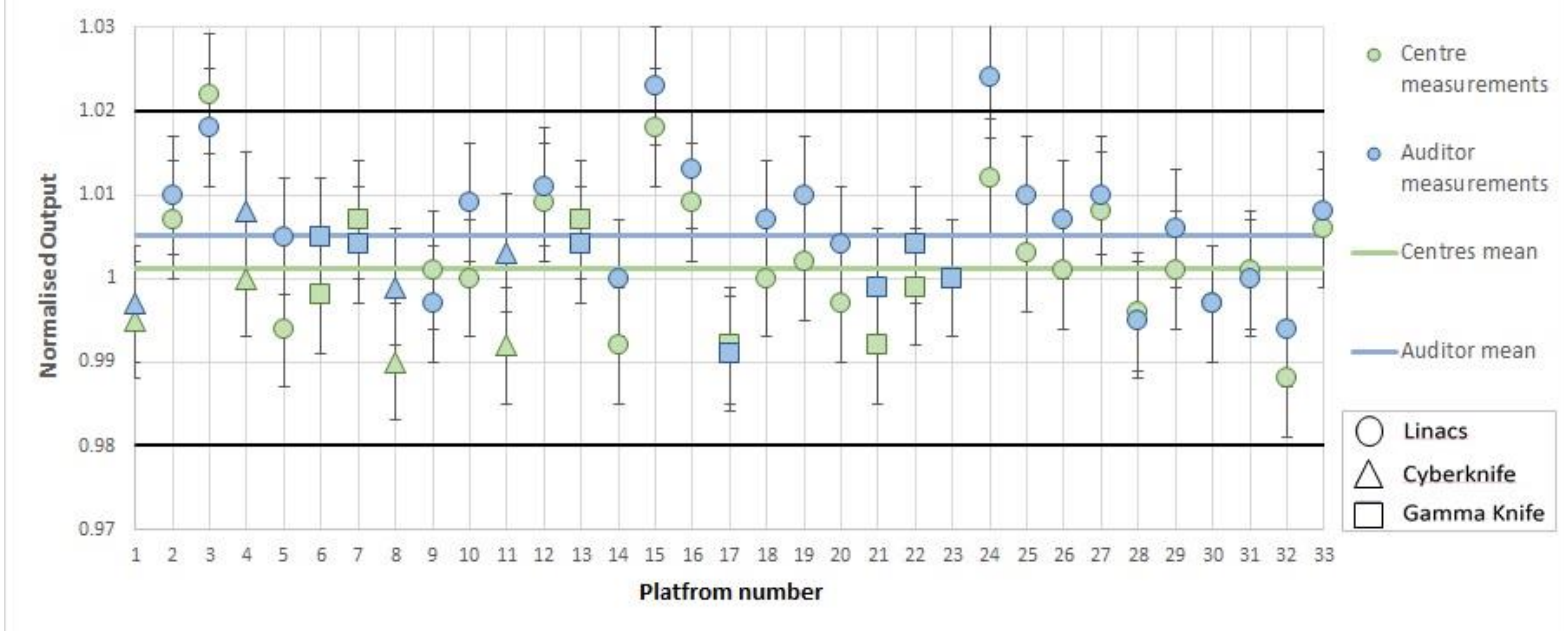

26

Figure 3: Output measurements in local reference conditions for the 33 platforms that participated in the audit. An uncertainty of $\pm 0.7 \%$ ( $k=1)$ is indicated by the error bars as a standard protocol based on the calibration certificate of the detector. The "acceptable" tolerances of $\pm 2 \%$ are indicated. 
Short title: Multi-institutional audit of dosimetric delivery in radiosurgery

\section{A. Percentage difference in the target - mean of 4 pellets Vs TPS}

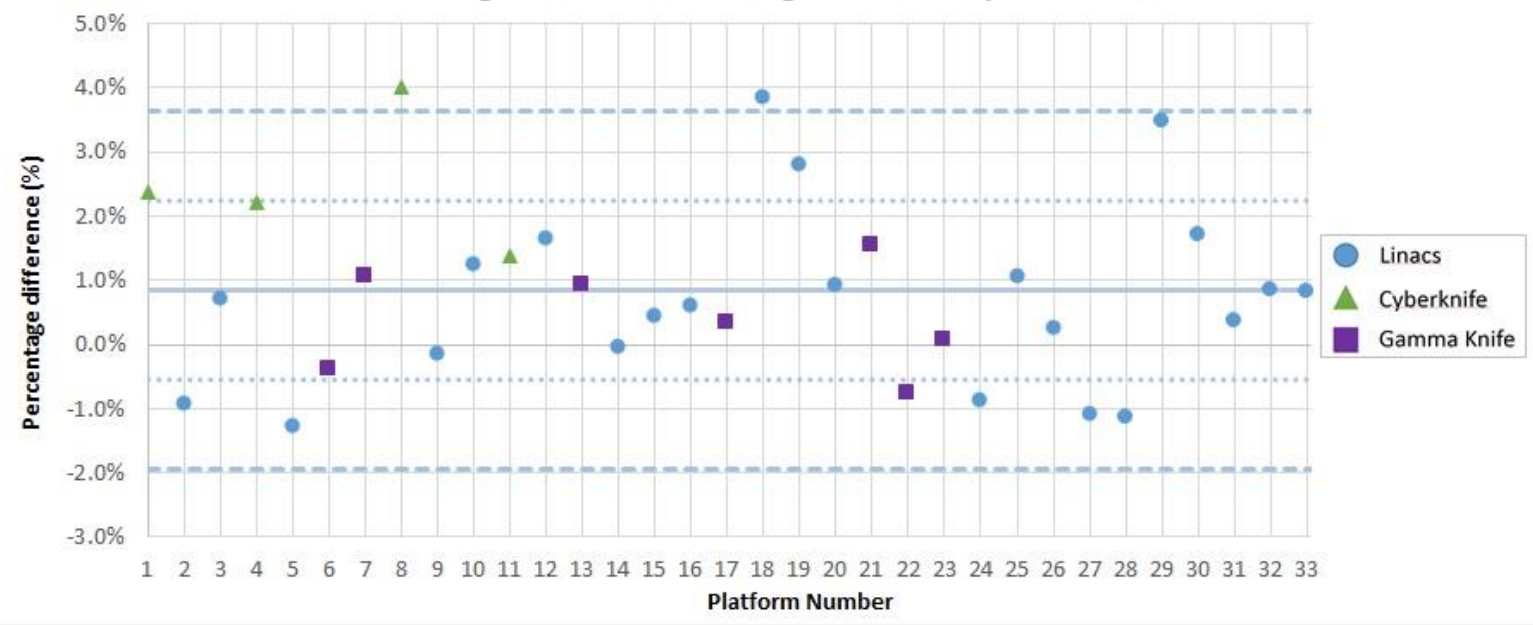

B. Percentage difference in the brainstem - mean of 4 alanine pellets Vs TPS (normalised to $12 \mathrm{~Gy}$-nominal brainstem tolerance dose)

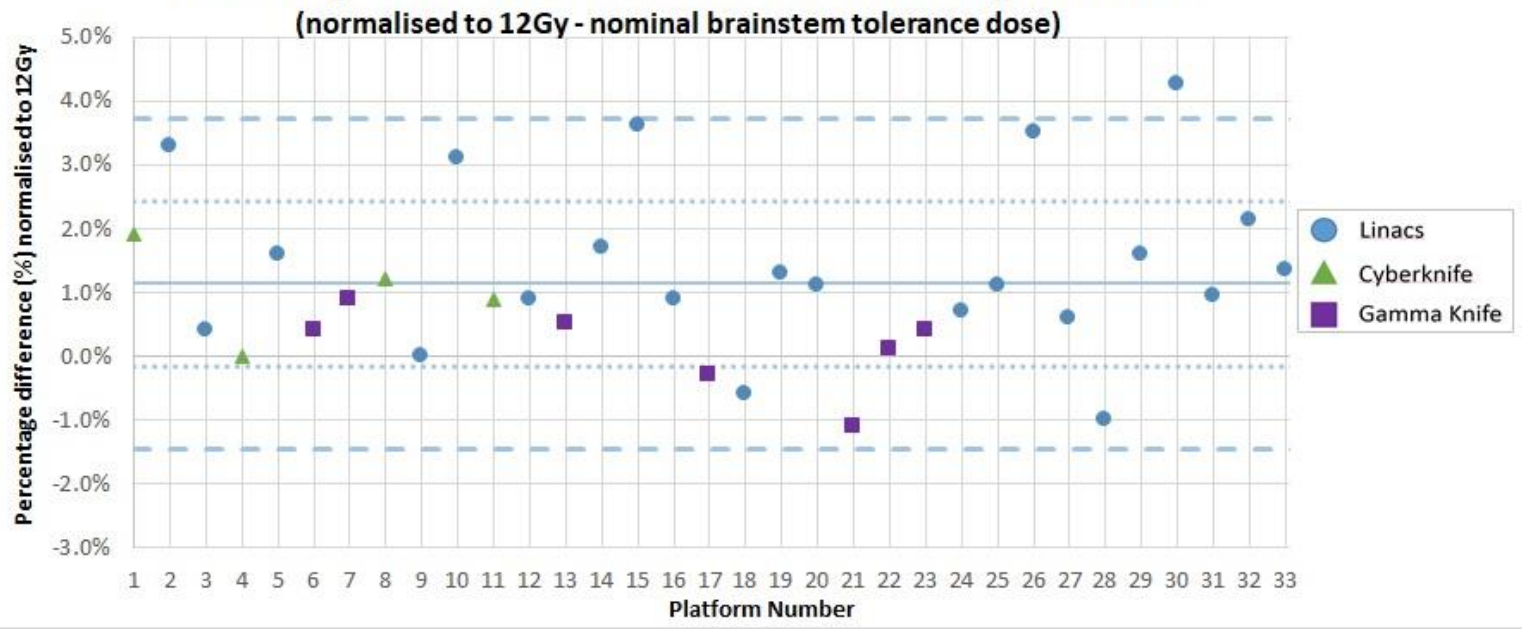

Figure 4: Alanine pellet measurements performed in the target (4A) and organ at risk (4B). Platform 646 groups are indicated in the legends. The mean for all centres is represented by the solid blue line, the dotted lines represent one standard deviation of the mean and the dashed lines represent two standard 648 deviations of the mean. 

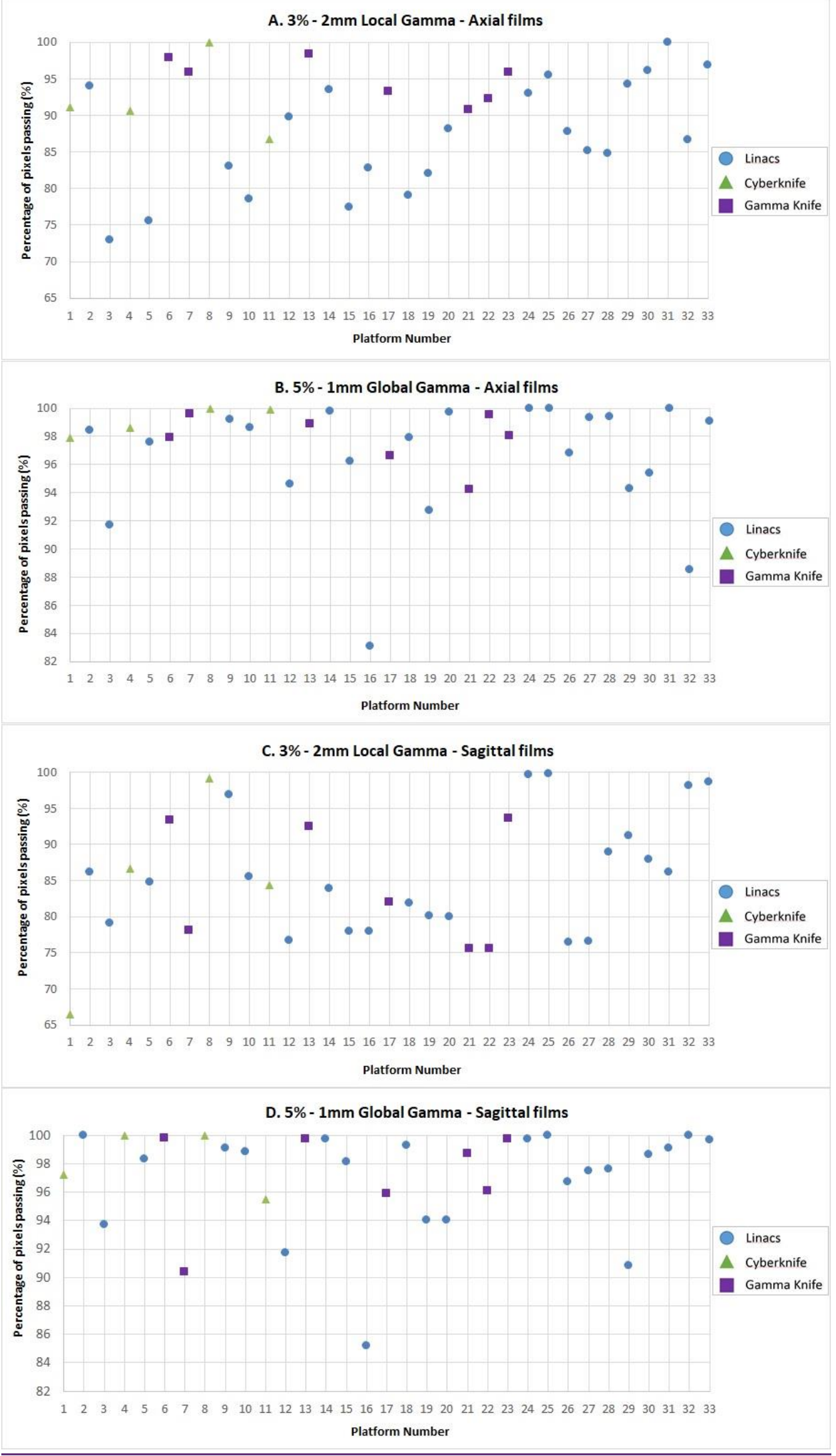

Figure 5: Axial (5A\&5B) and Sagittal (5C\&5D) film passing rates of the local gamma criterion of 3\% $2 \mathrm{~mm}$ and the global gamma criterion of $5 \%-1 \mathrm{~mm}$ for the 33 platforms that were assessed. 
Short title: Multi-institutional audit of dosimetric delivery in radiosurgery

1

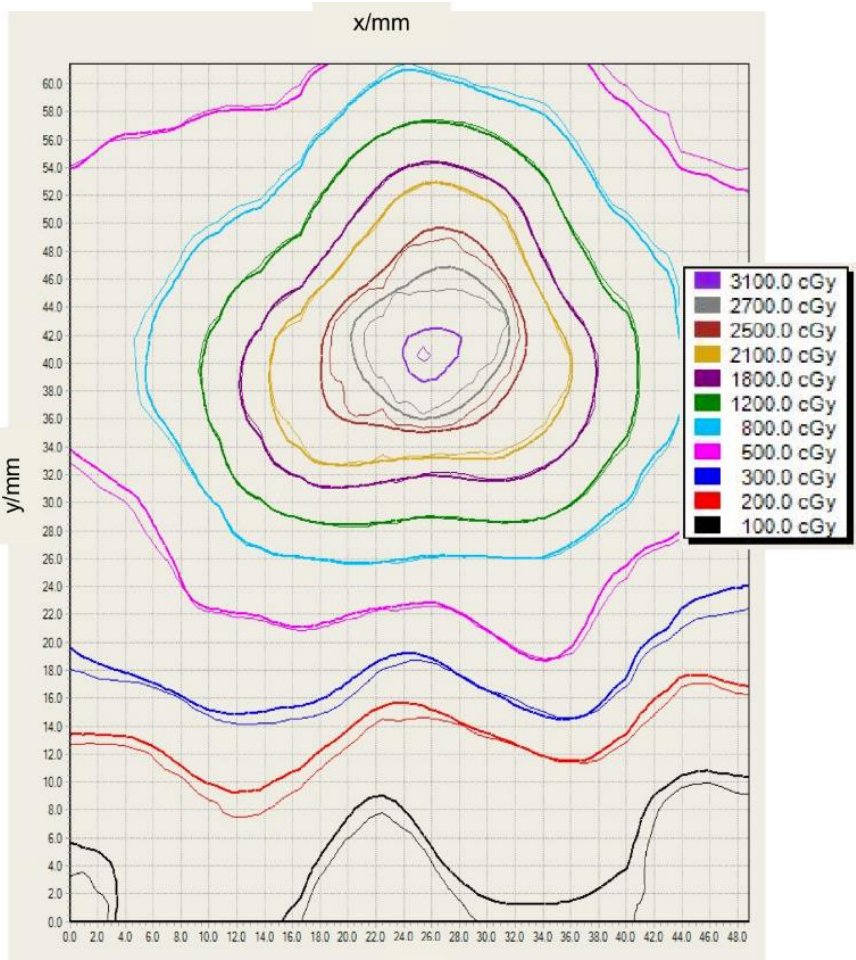

Axial

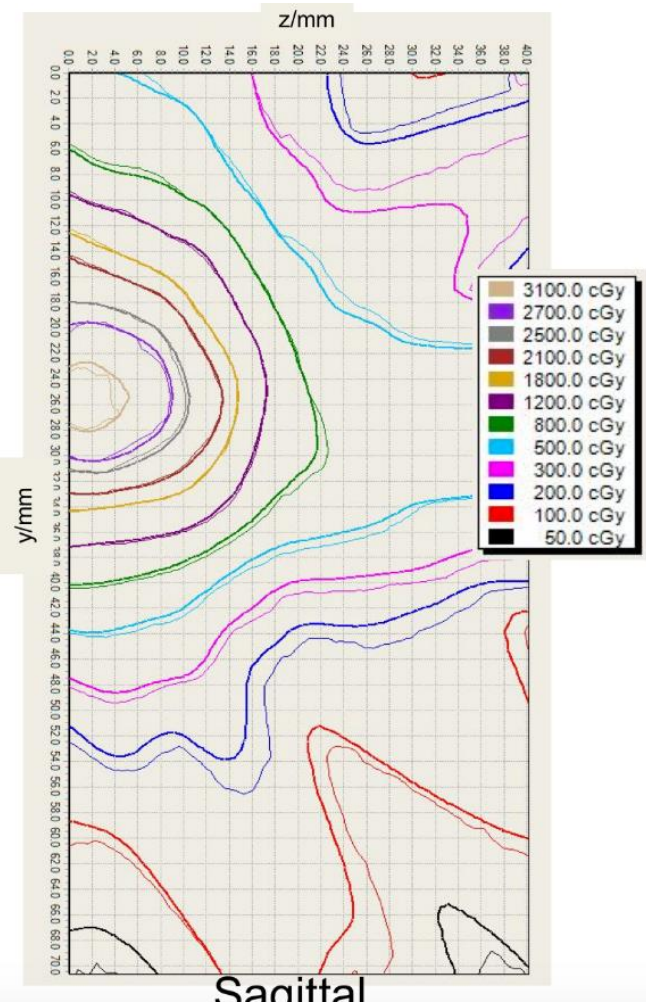

Sagittal

Supplementary Figure 1: Example of dose distribution comparisons between the film-measured doses (thin lines) and the treatment planning system- calculated doses (thick lines) for the axial and the sagittal films used. 

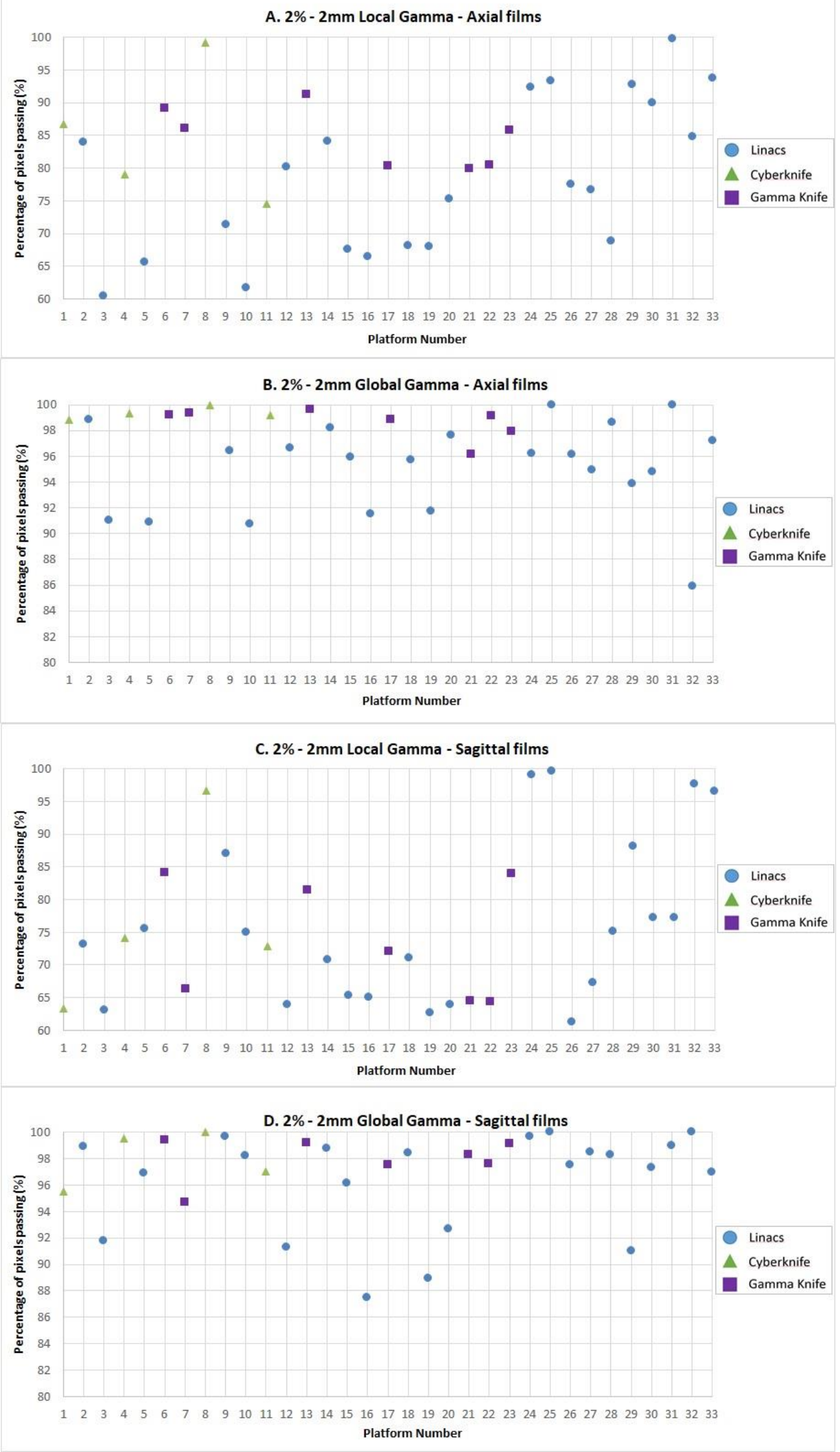

Supplementary Figure 2: Axial (A\&B) and Sagittal (C\&D) film passing rates of the local gamma and 695 the global gamma criteria of $2 \%-2 \mathrm{~mm}$ the 33 platforms that were assessed. 
Short title: Multi-institutional audit of dosimetric delivery in radiosurgery

1 Title: Multi-institutional dosimetric delivery assessment of intracranial stereotactic

4 radiosurgery on different treatment platforms

Keywords: Radiosurgery, Dosimetry, End-to-end, Audit, Anthropomorphic Phantom, Alanine, Radiochromic film

\section{Highlights:}

- A multi-institutional end-to-end assessment of radiosurgery dosimetric delivery was performed for 33 plans in 30 participating centres using a variety of treatment platforms.

- The comparison has highlighted the dosimetric consistency achievable with different delivery platforms.

- The need for standardisation in intracranial stereotactic radiosurgery is highlighted.

\section{Abstract:}

\section{Background and Purpose:}

Assessment of dosimetric accuracy of radiosurgery on different treatment platforms. Material and Methods:

Thirty-three single fraction treatment plans were assessed at thirty centres using an anthropomorphic head phantom with target and brainstem structures. The target being a single irregular shaped target, $\sim 8 c c, 10 \mathrm{~mm}$ from the brainstem. The phantom was "immobilised", scanned, planned and treated following the local protocols. EBT-XD films and alanine pellets were used to measure absolute dose, inside both the target and the brainstem, and compared with TPS predicted dose distributions.

\section{Results:}

PTV alanine measurements from gantry-based linacs showed a median percentage difference to the TPS of $0.65 \%$. Cyberknife (CK) had the highest median difference of $2.3 \%$ in comparison to the other platforms. GammaKnife (GK) showed the smallest median of $0.3 \%$. Similar trends were observed in the OAR with alanine measurements showing median percentage differences of $1.1 \%, 2.0 \%$ and $0.4 \%$, for gantry-based linacs, CK and GK respectively. All platforms showed comparable gamma passing rates between axial and sagittal films.

Conclusions:

This comparison has highlighted the dosimetric variation between measured and TPS calculated dose for each delivery platforms.. The results suggest that clinically acceptable agreement with the predicted dose distributions is achievable by all treatment delivery systems. 
Short title: Multi-institutional audit of dosimetric delivery in radiosurgery

\section{Introduction}

Stereotactic radiosurgery (SRS), was first developed in the 1950s [1] and has since evolved substantially. There are now several manufacturers that offer commercial solutions for delivering SRS and such treatments may be delivered by a Gamma Knife (GK) unit, a CyberKnife (CK) or a gantry-based linear accelerator (linac) system with stereotactic capabilities.

All radiotherapy practices should be subjected to appropriate quality assurance procedures, including regular quality control testing and independent external dosimetry audit $[2,3]$, to minimize potential errors in treatment delivery that can lead to clinical complications [4]. This is especially the case for SRS where a very high dose is delivered in only a single fraction, meaning an error cannot be mitigated in a subsequent fraction.

The multiple platforms which can be used for SRS are very different in terms of their technologies and techniques [5]. Furthermore, they have been, and are being, used very differently in terms of their dosimetric and clinical practices [6]. The categorisation of the various systems into Gamma Knife (GK) units, CyberKnife (CK) units or gantry-based linear accelerator (linac) systems with stereotactic capabilities is justified according to their broadly similar dosimetric and clinical practices [6]. The latter category includes systems which use cones or tertiary microMLCs and also includes Tomotherapy units, which use a fixed ring gantry and have no non-coplanar delivery capability.

This national study was undertaken to support an initiative in the UK to regulate the provision of cranial SRS services [7-9]. The participating centres were audited in an end-to-end test, incorporating local clinical procedures for immobilisation devices, CT-scanning, target contouring, treatment planning and treatment delivery. We evaluated the agreement between planned and delivered dose for each of the audited systems. It is acknowledged that the audited systems cover a wide range of delivery and ancillary systems, however such a study has the potential to benchmark what can be achieved with intracranial stereotactic surgery systems and therefore contribute data for use in setting tolerances for clinical trials and future external 
Short title: Multi-institutional audit of dosimetric delivery in radiosurgery

76
78

79

80

81

82

83

84

85

86

87

88

audits. Furthermore, the variations in clinical practice observed between these different platforms have been evaluated to assess the need for standardisation.

\section{Materials and Methods}

A comprehensive end-to-end test was developed based on an anthropomorphic head phantom, STEEV (Stereotactic End-to-End Verification, CIRS, Norfolk, VI, USA). The phantom was adapted to contain a single irregularly shaped target $(\sim 8 \mathrm{cc})$, $10 \mathrm{~mm}$ anterior to the brainstem, for treatment by the audited centre following their local protocol for brain metastasis to be treated in a single fraction [10]. A Computed Tomography (CT) scan of the phantom was sent in advance to the participating centres in DICOM format for volume contouring and pre-planning. This was followed by a visit to the centre where the phantom was scanned following the local protocol (CT only), with eight dummy alanine pellets and two dummy pieces of EBT-XD Gafchromic film (Ashland ISP Advanced Materials, NJ, USA) placed inside the phantom to mimic the detector positions. These were subsequently replaced with the real detectors prior to phantom treatment irradiation. A graphical representation showing the target (PTV), brainstem (OAR) and detector positions is included in Figure 1. The two CT scans were co-registered, and the pre-plan transferred to the local scan and finalised.

Thirty centres participated in the audit. In three centres two treatment platforms were assessed and hence thirty-three single fraction treatment plans were generated and delivered to the phantom. Table 1 provides details for each platform and plan that participated in the audit. Further details of the auditing protocol followed are given in Figure 2. 


\subsection{Reference beam output measurements}

A calibrated PTW 31010 semiflex ionisation chamber $(0.125 \mathrm{cc})$, traceable to a graphite calorimeter primary standard at the National Physical Laboratory (NPL, Teddington, UK) was used in all centres to perform reference beam output measurements. The chamber was placed in an auditor provided water-equivalent plastic material, using the centre's reference conditions, to measure the output in the machine specific reference field [11]. All such auditor measurements were corrected for temperature and pressure. Temperature and pressure were measured using independent auditor equipment. The measurements were performed in machinespecific reference conditions and deviations from agreement were expressed relative to the TPS-calculated value for dose in a geometric water equivalent phantom.

The reference beam output measurements were used to apply corrections to the alanine measurements of the clinical plan, to compensate for any daily output variation.

\subsection{Alanine measurements}

Two sets of four alanine pellets, each a disc of $5 \mathrm{~mm}$ diameter and $2.5 \mathrm{~mm}$ thickness, 127 were irradiated in each centre: one in the target and one in the brainstem. The 128 phantom temperature before and after each measurement was recorded and used to apply a temperature correction factor. All pellets were returned to NPL and were processed within one month of the audit visit, ensuring negligible fading [12]. The measured doses were compared to the mean dose calculated by the TPS for each pellet. Additionally, in order to account for positional uncertainties that can lead to large percentage differences between measured and predicted doses, mean doses for each pellet stack were compared with the TPS prediction for the complete stack.

The percentage difference between alanine and TPS for the OAR was normalised to the 12 Gy dose level, being the nominal brainstem tolerance dose used by many centres. This enabled a dosimetric comparison whilst assessing the plan quality in terms of overdose to the OAR.

Uncertainty on the alanine readout was taken to be $1.4 \%(\mathrm{k}=2)$ [12]. The deviations 140 from agreement were expressed relative to the TPS-calculated value. 
Alanine was considered as the primary detector for the audit measurements, as it has proven its efficacy in small fields and is provided by an established dosimetry service based at NPL [12].

\subsection{Film measurements}

The film response was calibrated in a conventional manner with ten EBT-XD film pieces exposed in the range of 0-40 Gy. The films were irradiated in a $10 \mathrm{~cm} \times 10$ $\mathrm{cm}$ field at $5 \mathrm{~cm}$ depth in water-equivalent plastic, with a nominal $6 \mathrm{MV}$ beam. The scanned pixel value as a function of dose was determined as the average pixel value in a $4 \times 4 \mathrm{~cm}$ region centred on the beam axis. Images were converted to dose maps using FilmQAPro® software (Ashland ISP Advanced Materials, NJ, USA) using the red-green-blue triple-channel dosimetry algorithm [13].

Two films (one sagittal and one axial) were placed inside the phantom and simultaneously irradiated at each centre. All films were returned to NPL and scanned at least 72 hours after exposure following an established film dosimetry protocol $[14,15]$ and good film dosimetry practice $[16,17]$ with the analysis performed on FilmQAPro. Film-dose linear scaling was applied using reference films at zero dose and $80 \%$ of the maximum anticipated dose from the treatment plan, which were scanned simultaneously with the test films. This approach mitigates the effects of post-exposure darkening and variations of the scanner response, and stabilizes the calibration (forced into agreement) at the reference dose levels [18]. The regions of interest used for the analysis were a $6 \times 5 \mathrm{~cm}$ rectangle for the axial films and a $7 \times 4$ $\mathrm{cm}$ rectangle for the sagittal films. The measured dose distributions were matched to TPS dose distributions by aligning the fiducial marker holes in the film with known coordinates in the TPS dose planes and then using the optimum shift algorithm, where small shifts were applied $\left(<1 \mathrm{~mm}\right.$ and $\left.<1^{\circ}\right)$ to optimise the gamma passing rate..

Gamma passing rates [19] were collected for a range of criteria (global and local) using the red colour channel with triple-channel-correction and with film-dose linear scaling corrections applied. The criteria selected were based on the following: data for $3 \%$ dose difference and $2 \mathrm{~mm}$ distance $(3 \% / 2 \mathrm{~mm})$ were collected as this criterion was commonly used by the participating linac users and $5 \% / 1 \mathrm{~mm}$ as this is more suitable for plans with steep gradients. Data for $2 \% / 2 \mathrm{~mm}$ was also analysed as recommended 
by AAPM TG 135 [20]. A minimum cut-off threshold of 2 Gy was applied to remove low dose areas of higher uncertainty from the analysis. This absolute value was used as an alternative to a relative value, due to the large variation of prescription doses and maximum doses delivered by the audit participants.

\subsection{Statistical analysis}

A Kruskal-Wallis test was performed to detect any significant differences in median values between the three platforms, followed by the Bonferroni-type multiple comparison to establish the hierarchy/significant differences between different pairs.

\section{Results}

Reference output measurements were performed independently by both the host centre and the auditors using their respective equipment and these are presented in Figure 3. All measurements from the auditors and the centres were within $\pm 2.4 \%$ of the dose calculated by the TPS, ranging from $-1.0 \%$ to $+2.4 \%$. The mean difference between auditor measurement and host centre measurement (audit/host) was $+0.5 \%$, with a maximum difference of $+1.2 \%$. The gantry based linac group spread in the output measurements was $3.2 \%$, ranging from $-0.8 \%$ to $+2.4 \%$, with a median of $0.35 \%$. CKs had a spread of $1.8 \%$, ranging from $-1.0 \%$ to $+0.8 \%$, with a median of $0.85 \%$ and GKs had a of spread, $1.6 \%$, ranging from $-0.9 \%$ to $+0.7 \%$, with a median of $0.0 \%$. No statistical differences were seen between the platforms.

The differences between the measured and calculated (measured/calculated) doses for the stack of 4 alanine pellets ranged from $-1.3 \%$ to $+4.0 \%$ for the PTV (Figure $4 \mathrm{~A}$ ). The gantry based linacs showed a spread in percentage difference of $5.2 \%$ (from $1.3 \%$ to $+3.9 \%$ ) with a median of $0.65 \%$. GKs showed a spread of $2.4 \%$ (from $-0.8 \%$ to $+1.5 \%$ ), with a median percentage difference of $+0.3 \%$. CK measurements had a spread of $2.6 \%$ (from $+1.4 \%$ to $+4 \%$ ), with a median of $+2.3 \%$, which in comparison with the gantry based linac and GK groups were statistically higher with $p$ values of 0.045 and 0.039 respectively. Individual pellet measurements in the target showed differences of up to $14.1 \%, 4.2 \%$ and $5.9 \%$ (for GK, CK and linac respectively) when 
compared to the TPS-predicted mean dose in their individual contoured pellet structure.

Similar trends were observed in the comparison of OAR alanine pellet measurements to those observed in target alanine pellet measurements, ranging from $-1.1 \%$ to $+4.3 \%$ (Figure 4B). Gantry-based linac measurements showed a spread at 4.6\% ranging from $-1 \%$ to $+3.6 \%$ with a median of $+1.2 \%$. CK measurements ranged between $0.0 \%$ to $+1.9 \%$ with a median of $+1.1 \%$. GKs had a spread of $2.0 \%$ ranging from $-1.1 \%$ to $+0.9 \%$ and a median of $+0.4 \%$. Here the only significance was in the gantry-based linac group being higher than the GK group with a $p$ value of 0.047 .

TPS-predicted doses for the alanine pellets in the OAR ranged from 0.3 Gy up to 7.5 Gy. Figure 4 shows the percentage difference between the mean dose measured by OAR alanine pellets with the TPS-predicted mean dose, normalised to $12.0 \mathrm{~Gy}$.

The results for axial and sagittal films (example shown in Supplementary Figure 1) showed consistency in the gamma passing rates achieved. The gamma passing rates for $3 \% / 2 \mathrm{~mm}$ local gamma and 5\% / 1mm global gamma are shown in Figure 5.

Higher passing rates for all films were observed for global than for local gamma criteria. For the $3 \% / 2 \mathrm{~mm}$ local gamma criterion, all but two films showed passing rates above $75 \%$. The CK, GK and gantry-based linac groups had median passing rates of $88.7,92.8$ and 85.5 respectively, showing no statistical differences between them. For the $5 \% / 1 \mathrm{~mm}$ global gamma criterion, all but 3 films showed passing rates above 90\% (see Figure 5). Here the CK, GK and gantry-based linac groups had median passing rates of $99.3,98.4,98.3$ respectively, again showing no statistical differences between them. For the $2 \% / 2 \mathrm{~mm}$ global gamma criterion, all but 3 films showed passing rates above 90\% (see Supplementary Figure 2). Here the CK, GK and gantry-based linac groups had median passing rates of 99.3, 99.0, 96.6 respectively, with a $p$ value of 0.028 between the GK and the gantry-based linac groups, all other comparisons being non-significant. 
When the regions of interest used for the gamma analysis were reduced to smaller areas to include the target region only, passing rates improved substantially for all centres, showing very good agreement (>95\%) between TPS-predicted and delivered dose distributions. The majority of failed pixels for all films analysed were found to be outside the target, between the 2 Gy (threshold level) and the 12 Gy isodose line.

\section{Discussion}

The reference output measurements performed in the standard conditions for each platform at all centres were within $\pm 2.4 \%$ of the expected dose, well within the $\pm 3 \%$ recommendation of IPEM report 81 [21]. Moreover, the results seen in this study are consistent with the results reported by the NPL over 20 years of reference audits [22]. The differences from expected dose seen in the GK and CK groups were comparable, and much smaller than those seen in gantry-based linacs. This could be related to these platforms having more predictable output in reference conditions, due to having a more straight forward design. The ${ }^{60} \mathrm{Co}$ sources in the GK have a predictable decay, which is reflected in the good agreement seen in these measurements and the tighter tolerances of 1.0 - 1.5\% deviation used by GK centres. The compact single energy linear accelerator of the CK may be the reason for the reduced fluctuations observed in output measurements, compared to multi-energy and multi-modality conventional gantry-based linacs which comprise the majority of units within this category. The systematic difference between the auditor and the centres $(+0.5 \%)$ may be partly explained by the instruments used. While the auditors used a $0.125 \mathrm{cc}$ ionisation chamber for all measurements, the centres used a range of different detectors, which in most cases had a larger sensitive volume. Therefore, a small degree of volume averaging can be attributed to the difference observed, especially since 15 out of 33 participating platforms used Flattening Filter Free (FFF) type beams. However, further measurements need to be performed to fully explain this difference.

Overall, good agreement was observed between alanine and TPS, with three centres falling outside (greater than) two standard deviations of the mean (two centres in the target dose measurements and one in the OAR measurements). Although there were some statistical differences between the groups these had $p$ values which were only 
just less than 0.05 and were not consistent across the measurement methods and PTV/OAR, hence no strong statistical conclusions can be drawn about each platform.

The gantry-based linac group was seen to have the largest spread in percentage differences compared to $\mathrm{CK}$ and GK, but with a relatively good overall agreement with the TPS. The reasons behind this spread are most likely attributed to the diversity of techniques, platforms, beam energies, TPSs, calculation algorithms, clinician preferences and influences from the local radiotherapy practices. In comparison, the CK and GK groups had almost identical settings and practices within their subgroups. It is also possible that the variations in the commissioning methodologies used for gantry-based linacs, especially with regards to the dosimeters used for the measurement of small radiation fields, has an impact on the accuracy of dose calculation. Although numerous studies have been conducted on appropriate detectors for small field applications [23-28], there has been a lack of international guidelines until the recently published IAEA TRS 483 [29]. It is expected that these new guidelines will improve the standardisation of commissioning methodologies.

282 CK measurements in the target, showed that the TPS with Raytracing calculation 283 algorithm under-estimated the dose in all four centres visited. This finding is in 284 agreement with another study utilising the same alanine service for target dose 285 measurements in CK plans [30].

286 All GK centres used a TMR10 algorithm that does not account for density 287 inhomogeneities and assumes water density within a CT-generated or depth-helmet 288 measured skull contour. A recent study investigating the GK convolution algorithm 289 (employs density corrections) in comparison to the TMR10, showed a 6\% difference 290 between the two where $1.5 \%$ of this was attributed to depth helmet measurements 291 [31]. Our alanine measurements showed good agreement with the TPS and suggest 292 that these sources of error do not contribute significantly to dosimetric inaccuracies. 293 Further work investigating this convolution algorithm may be required to evaluate its accuracy before it is used clinically.

Alanine measurements in the OAR were performed along a steep dose gradient where any positional uncertainties may be expressed as large dose differences. Doses to the OAR pellets ranged by up to an order of magnitude, caused by individual planning priorities and protocols used by participating centres. As some centres delivered very 
low doses to this region the reported doses were normalised to $12 \mathrm{~Gy}$ (a nominal brainstem tolerance dose value used by many centres) in order to provide a useful measure of relative accuracy to the centres with different approaches. There was also a higher uncertainty in the lower dose measurements due to lower signal to noise ratios in the alanine readout.

304 The gamma passing rates showed clinically acceptable agreement between the film305 measured dose and the treatment planning system calculated dose distributions for 306 both sagittal and axial films [32]. All treatment modalities showed comparable variations in passing rates between the centres assessed and the passing rates alone do not suggest significant differences between the different platforms. Other studies have suggested that gamma index analysis is non-ideal for direct comparison in multiinstitution assessments due to inherent differences in the dose distributions, particularly in dose gradient and maximum doses [32-34]. Local Gamma criteria may favour linac centres in which the dose gradient could be less steep than GK and CK, and Global Gamma criteria may favour GK centres in which the maximum (normalisation) dose is higher and the dose gradients are steeper. Whilst the methodology for film analysis employed in this study was designed to diminish sensitivities to different dose distributions it is impossible to achieve this with gamma index analysis. Gamma passing rates are also sensitive to the position of the film relative to the dose plan, the position of the region of interest used for the analysis and the 2 Gy threshold levels applied. An analysis method that is less sensitive to these dose distribution differences could be preferable, enabling a more reliable direct comparison between competing plans. Future studies of this nature must develop novel methodologies to enable more meaningful and clinically relevant comparisons, such as 3D dosimetry and improvements in the analysis of dose distributions moving towards a DVH-based assessment. The use of dose-plane-histograms (analogous to dose-volume histograms (DVH) in a single plane) may provide a more clinically relevant analysis $[35,36]$. Despite its pitfalls, the gamma analysis method used, enabled quantification of the dose shaping abilities of all SRS platforms active in the UK. The results showed clinically acceptable dosimetric performance by all platforms, although noticeable dosimetric differences were apparent outside the target volume, which are unlikely to be clinically relevant. These dosimetric inaccuracies, seen in most centres, are related to the TPS's limitations in simulating 
out of field doses and typically resulted in underestimation of doses to the OAR, as found by other studies [37]. Another study conducting film-based end-to-end tests in CK plans recorded higher gamma passing rates (>90\%) for the criterion of $2 \%-2 \mathrm{~mm}$ local gamma [38], compared to the median passing rates seen in this study of 76.8, 81.0 and $75.1 \%$ for CK, GK and gantry-based linacs respectively. The differences seen are explained by the higher $50 \%$ dose threshold used in that study that excludes the low doses included in our film analysis.

While other multi-platform assessments have been conducted on stereotactic applications [30,39,40] only a few have been performed specifically for SRS [41]. This study is novel in the diversity of treatment platforms included and the advanced dosimetry methods employed. In this study, thirty-three treatment plans with differing planning approaches were generated, all for the same realistic patient scenario of a single metastatic lesion located anterior to the brainstem. Aside from these planning differences, some subtle differences were observed in the accuracy of the measured dose distributions. However, the most influential and clinically relevant variation observed in the protocols assessed, was found in prescription practices (Table 1), highlighting the potential need for standardisation.

Although a statistical analysis was performed, the small numbers in each group means a larger population would be needed for a more robust analysis. However, this was a national study and a larger study could only be realised at a multi-national level. Such a larger study may also reveal more subtle differences in the individual approaches followed in each centre. Another limitation of this work is the lack of incorporation of MRI in the end-to-end assessment, which is an integral step in intracranial SRS.

Currently, the assessment of dosimetric deliveries in a multi-platform, multi-centre SRS setting with individual planning priorities remains a challenge. However, independent dosimetric assessments as presented in this study are important interventions which have a crucial role in ensuring accurate dose delivery to patients. Moreover, when data from multiple centres is pooled together, it enables participants to benchmark their services against the rest of their community, assess their safety, 
evaluate their practices and consider improvements to their service. As stated the basis of this audit was to support an initiative in the UK to regulate the provision of cranial SRS services. Consistent dosimetry has been recognised as essential in the evaluation of outcomes from those centres commissioned to provide such clinical services. Overall, there was a smaller spread of data seen in the CK and GK groups, however each had some statistically significant differences with the other platforms.

Future multicentre SRS studies may benefit from some standardisation and consensus of practice.

\section{Acknowledgments}

Funding for PhD studies and partial travel costs - Engineering \& Physical Sciences Research Council (EPSRCEP/J500094), Technical support (Ilias Billas, Michael Homer, Ana Subiel, Simon Duane, Sebastian Galer), Alanine analysis (Gavin Cox, David Crossley), Audit support (Elizabeth Miles, Rada Zotova), Pilot audit support (Martyn Gilmore, Phillip Cooper, Kelvin Hiscoke).

\section{References}

[1] Leksell L. The stereotaxic method and radiosurgery of the brain. Acta Chir Scand 1951;102:316-9.

[2] Clark $\mathrm{CH}$, Jornet $\mathrm{N}$, Muren LP. The role of dosimetry audit in achieving high quality radiotherapy 2018. https://doi.org/10.1016/j.phro.2018.03.009.

[3] Clark CH, Ga Aird E, Bolton S, Miles EA, Nisbet A, Snaith JA, et al. Radiotherapy dosimetry audit: Three decades of improving standards and accuracy in UK clinical practice and trials. Br J Radiol 2015;88. https://doi.org/10.1259/bjr.20150251.

[4] Briggs G, Ebdon-Jackson S, Erridge SC, Graveling M, Hood S, McKenzie A, et al. Towards Safer Radiotherapy. R Coll Radiol Soc Coll Radiogr Inst Phys Eng Med Natl Patient Saf Agency Br Inst Radiol 2008:85. https://doi.org/978-1905034-25-3.

[5] Kocher M, Wittig A, Piroth MD, Treuer H, Seegenschmiedt H, Ruge M, et al. 
Stereotactic radiosurgery for treatment of brain metastases: A report of the DEGRO Working Group on Stereotactic Radiotherapy. Strahlentherapie Und Onkol 2014;190:521-32. https://doi.org/10.1007/s00066-014-0648-7.

[6] Dimitriadis A, Kirkby KJ, Nisbet A, Clark CH. Current Status of Cranial Stereotactic Radiosurgery in the UK. Br J Radiol 2016;89:20150452. https://doi.org/10.1259/bjr.20150452.

[7] NHS England. Stereotactic Radiosurgery/ Stereotactic Radiotherapy Needs Assessment and Service Review Consultation Report. 2015.

[8] NHS England. Service Specifications: Stereotactic radiosurgery and stereotactic radiotherapy (Intracranial) (All ages). London: 2016.

[9] Eaton DJ, Lee J, Paddick I. Stereotactic radiosurgery for multiple brain metastases: Results of multicenter benchmark planning studies. Pract Radiat Oncol 2018;0. https://doi.org/10.1016/..prro.2017.12.011.

[10] Dimitriadis A, Palmer AL, Thomas RAS, Nisbet A, Clark CH. Adaptation and validation of a commercial head phantom for cranial radiosurgery dosimetry end-to-end audit. Br J Radiol 2017;90:20170053. https://doi.org/10.1259/bjr.20170053.

[11] Alfonso R, Andreo P, Capote R, Huq MS, Kilby W, Kjäll P, et al. A new formalism for reference dosimetry of small and nonstandard fields. Med Phys 2008;35:5179-86. https://doi.org/10.1118/1.3005481.

[12] Sharpe PHG, Sephton JP. Therapy level alanine dosimetry at the NPL. Proc. 216th PTB Semin. Alanine Dosim. Clin. Appl. PTB-Dos-51, PTB, Braunschweig, 2006.

[13] Micke A, Lewis DF, Yu X. Multichannel film dosimetry with nonuniformity correction. Med Phys 2011;38:2523-34. https://doi.org/10.1118/1.3611636.

[14] Palmer AL, Dimitriadis A, Nisbet A, Clark CH. Evaluation of Gafchromic EBTXD film, with comparison to EBT3 film, and application in high dose radiotherapy verification. Phys Med Biol 2015;60:8741-52. https://doi.org/10.1088/0031-9155/60/22/8741.

[15] Dimitriadis A. Assessing the dosimetric and geometric accuracy of stereotactic radiosurgery. University of Surrey, 2017.

[16] Niroomand-Rad A, Blackwell CR, Coursey BM, Gall KP, Galvin JM, McLaughlin WL, et al. Radiochromic film dosimetry: Recommendations of AAPM Radiation Therapy Committee Task Group 5. Med Phys 1998;25:2093115. https://doi.org/10.1118/1.598869.

[17] Palmer A, Bradley D, Nisbet A. Evaluation and mitigation of potential errors in radiochromic film dosimetry due to film curvature at scanning. J Appl Clin Med 2015;16:425-31.

[18] Lewis D, Micke A, Yu X, Chan MF. An efficient protocol for radiochromic film dosimetry combining calibration and measurement in a single scan. Med Phys 2012;39:6339-50. https://doi.org/10.1118/1.4754797.

[19] Low DA, Harms WB, Mutic S, Purdy JA. A technique for the quantitative evaluation of dose distributions. Med Phys 1998;25:656-61. https://doi.org/10.1118/1.598248.

[20] Dieterich S, Cavedon C, Chuang CF, Cohen AB, Garrett JA, Lee CL, et al. Report of AAPM TG 135: Quality assurance for robotic radiosurgery. Med Phys 2011;38:2914-36. https://doi.org/10.1118/1.3579139.

[21] Lillicrap SC. Physics Aspects of Quality Control in Radiotherapy (Report No. 81). Phys Med Biol 2000;45:815-815. https://doi.org/10.1088/0031$9155 / 45 / 3 / 501$. 
[22] Thomas RAS, Bolt MA, Bass G, Nutbrown R, Chen T, Nisbet A, et al. Radiotherapy reference dose audit in the United Kingdom by the National Physical Laboratory: 20 years of consistency and improvements. Phys Imaging Radiat Oncol 2017;3:21-7. https://doi.org/10.1016/j.phro.2017.07.005.

[23] Silvestre I, Dimitriadis A, Subiel A. Relative output factors for TPS beam data acquisition with emphasis on small fields. Comparison of multiple detectors and multiple approaches. Rev Latinoam Fis Medica - ISSN 2413-9904 2017;3.

[24] Dimitriadis A, Patallo Silvestre I, Billas I, Duane S, Nisbet A, Clark CH, et al. Characterisation of a plastic scintillation detector to be used in a multicentre stereotactic radiosurgery audit. Radiat Phys Chem 2017:0-1. https://doi.org/10.1016/j.radphyschem.2017.02.023.

[25] Bassinet C, Huet C, Derreumaux S, Brunet G, Chéa M, Baumann M, et al. Small fields output factors measurements and correction factors determination for several detectors for a CyberKnife $\AA$ and linear accelerators equipped with microMLC and circular cones. Med Phys 2013;40:071725. https://doi.org/10.1118/1.4811139.

[26] Kamio Y, Bouchard $\mathrm{H}$. Correction-less dosimetry of nonstandard photon fields: A new criterion to determine the usability of radiation detectors. Phys Med Biol 2014;59:4973-5002. https://doi.org/10.1088/0031-9155/59/17/4973.

[27] Russo S, Masi L, Francescon P, Frassanito MC, Fumagalli ML, Marinelli M, et al. Multicenter evaluation of a synthetic single-crystal diamond detector for CyberKnife small field size output factors. Phys Medica 2016. https://doi.org/10.1016/j.ejmp.2016.03.005.

[28] Pimpinella M, Ciancaglioni I, Consorti R, Venanzio C Di, Guerra a S, Petrucci a, et al. A synthetic diamond detector as transfer dosimeter for $\mathrm{D}$ w measurements in photon beams with small field sizes. Metrologia 2012;49:S207-10. https://doi.org/10.1088/0026-1394/49/5/S207.

[29] Palmans H, Andreo P, Huq S, Seuntjens J. Dosimetry of small static fields used in external beam radiotherapy: An IAEA-AAPM International Code of Practice for reference and relative dose determination. Technical Report Series No. 483. laea Trs483 2017.

[30] Distefano G, Lee J, Jafari S, Gouldstone C, Baker C, Mayles H, et al. A national dosimetry audit for stereotactic ablative radiotherapy in lung. Radiother Oncol:406-10. https://doi.org/10.1016/j.radonc.2016.12.016.

[31] Rojas-villabona A, Kitchen N, Paddick I. Investigation of dosimetric differences between the TMR 10 and convolution algorithm for Gamma Knife stereotactic radiosurgery. J Appl Clin Med Phys 2016;17:1-13.

[32] Miften M, Olch A, Mihailidis D, Moran J, Pawlicki T, Molineu A, et al. Tolerance limits and methodologies for IMRT measurement-based verification QA:

Recommendations of AAPM Task Group No. 218. Med Phys 2018;45:e53-83. https://doi.org/10.1002/mp.12810.

[33] Ezzell GA, Burmeister JW, Dogan N, LoSasso TJ, Mechalakos JG, Mihailidis $\mathrm{D}$, et al. IMRT commissioning: Multiple institution planning and dosimetry comparisons, a report from AAPM Task Group 119. Med Phys 2009;36:535973. https://doi.org/10.1118/1.3238104.

[34] Hussein M, Clark CHH, Nisbet A. Challenges in calculation of the gamma index in radiotherapy - Towards good practice. Phys Medica 2017;36:1-11. https://doi.org/10.1016/j.ejmp.2017.03.001.

[35] Palmer AL, Nash D, Kearton JR, Jafari SM, Muscat S. A multicentre "end to end" dosimetry audit of motion management (4DCT-defined motion envelope) 
in radiotherapy. Radiother Oncol 2017;125:453-8.

https://doi.org/10.1016/j.radonc.2017.09.033.

[36] Sothmann T, Blanck O, Poels K, Werner R, Gauer T. Real time tracking in liver SBRT: Comparison of CyberKnife and Vero by planning structure-based $\gamma^{-}$ evaluation and dose-area-histograms. Phys Med Biol 2016;61:1677-91. https://doi.org/10.1088/0031-9155/61/4/1677.

[37] Kry SF, Bednarz B, Howell RM, Dauer L, Followill D, Klein E, et al. AAPM TG 158: Measurement and calculation of doses outside the treated volume from external-beam radiation therapy. Med Phys 2017;44:e391-429. https://doi.org/10.1002/mp.12462.

[38] Blanck O, Masi L, Damme M-C, Hildebrandt G, Dunst J, Siebert F-A, et al. Film-based delivery quality assurance for robotic radiosurgery: Commissioning and validation. Phys Medica 2015;31:476-83. https://doi.org/10.1016/j.ejmp.2015.05.001.

[39] Lambrecht M, Melidis C, Sonke J-J, Adebahr S, Boellaard R, Verheij M, et al. Lungtech, a phase II EORTC trial of SBRT for centrally located lung tumours a clinical physics perspective. Radiat Oncol 2016;11:7. https://doi.org/10.1186/s13014-015-0567-5.

[40] Kron T, Chesson B, Hardcastle N, Crain M, Clements N, Burns M, et al. Credentialing of radiotherapy centres in Australasia for TROG 09.02 (Chisel), a Phase III clinical trial on stereotactic ablative body radiotherapy of early stage lung cancer. Br J Radiol 2018:20170737. https://doi.org/10.1259/bjr.20170737.

[41] Seravalli E, Van Haaren PMA, Van Der Toorn PP, Hurkmans CW. Intercranial stereotactic RT A comprehensive evaluation of treatment accuracy, including end-to-end tests and clinical data, applied to intracranial stereotactic radiotherapy. Radiother Oncol 2015;116:131-8. https://doi.org/10.1016/j.radonc.2015.06.004. 


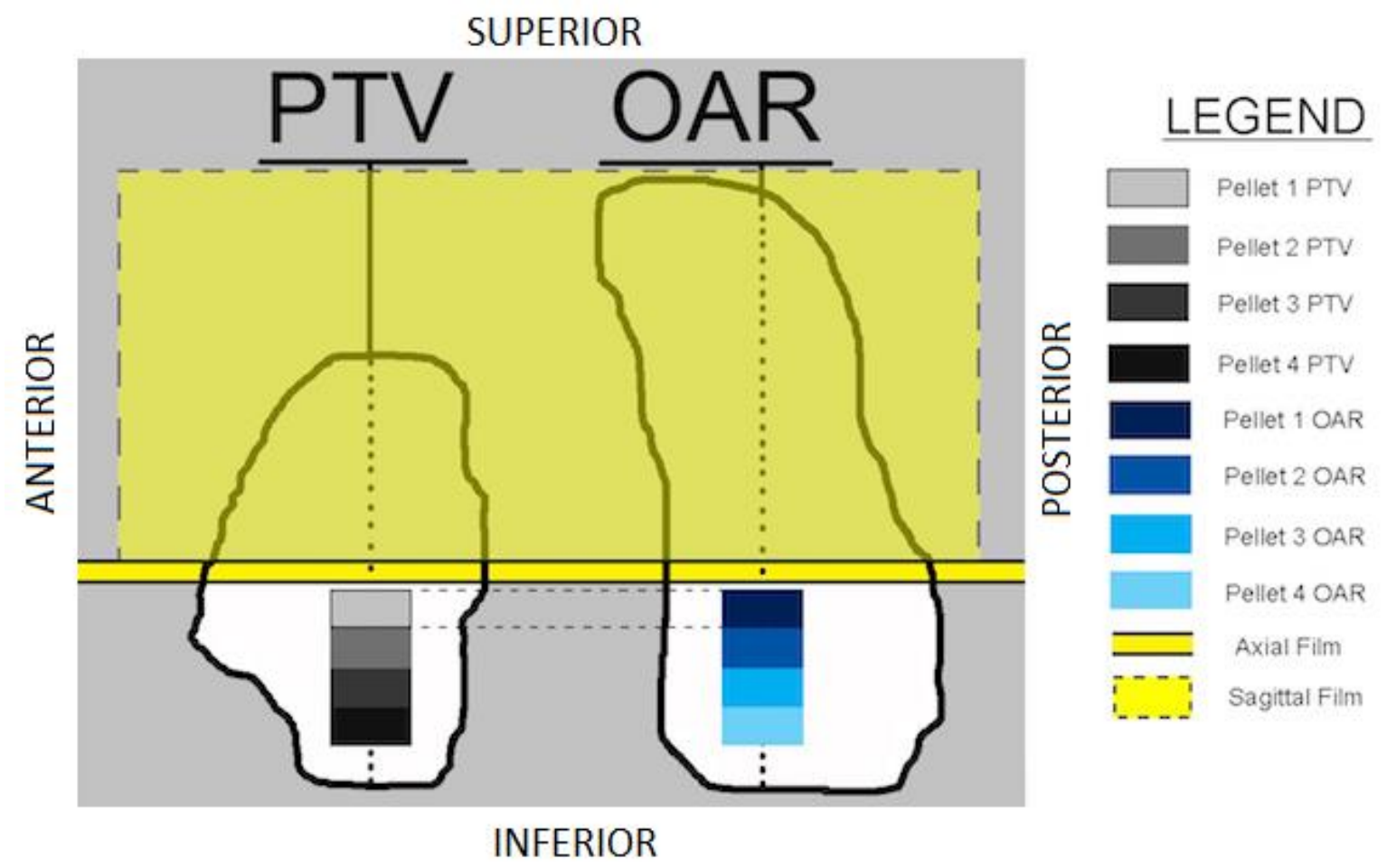

Figure 1: Schematic representation of a sagittal view through the centre of the phantom, showing the positions of the detectors in relation to the target and brainstem structures. 
Short title: Multi-institutional audit of dosimetric delivery in radiosurgery

1

\begin{tabular}{|c|c|c|c|c|c|c|c|c|c|c|}
\hline $\begin{array}{l}\text { Platfor } \\
\text { m No. }\end{array}$ & Platform & Energy & Technique & $\begin{array}{l}\text { Coplanar } \\
\text { / Non- } \\
\text { coplanar }\end{array}$ & TPS & $\begin{array}{c}\text { Dose } \\
\text { Calculation } \\
\text { Algorithm }\end{array}$ & $\begin{array}{c}\text { Density } \\
\text { Heterog. } \\
\text { Correction }\end{array}$ & $\begin{array}{l}\text { Peripheral } \\
\text { Prescr. } \\
\text { Dose (Gy) }\end{array}$ & $\begin{array}{l}\text { Max } \\
\text { Dose } \\
\text { (Gy) }\end{array}$ & $\begin{array}{c}\text { Pr Iso. } \\
\text { Relative to } \\
\text { Dmax (\%) }\end{array}$ \\
\hline 1 & $\begin{array}{c}\text { VRN } \\
\text { TrueBeam } \\
\text { STx } \\
\end{array}$ & 10MV FFF & 4DCA & $\mathrm{NC}$ & BL iPlan & $\begin{array}{l}\text { Pencil } \\
\text { Beam }\end{array}$ & Yes & 21.0 & 26.4 & $80 \%$ \\
\hline 2 & BL Novalis Tx & $6 \mathrm{MV}$ & $9 \mathrm{FF}$ & $\mathrm{NC}$ & BL iPlan & $\begin{array}{l}\text { Pencil } \\
\text { Beam }\end{array}$ & Yes & 17.5 & 22.7 & $77 \%$ \\
\hline 3 & VRN 2100x & $6 \mathrm{MV}$ & $4 \mathrm{CCA}$ & $\mathrm{NC}$ & BL iPlan & $\begin{array}{c}\text { Circular } \\
\text { Cone }\end{array}$ & Yes & 18.0 & 23.6 & $76 \%$ \\
\hline 4 & ELK Synergy & $6 \mathrm{MV}$ & 5VMAT & $\mathrm{NC}$ & Pinnacle & Convolution & Yes & 18.0 & 22.9 & $79 \%$ \\
\hline 5 & $\begin{array}{c}\text { ACC } \\
\text { Tomotherapy }\end{array}$ & $6 \mathrm{MV}$ FFF & $\begin{array}{l}\text { Tomo } \\
\text { Therapy }\end{array}$ & C & $\begin{array}{l}\text { Tomo } \\
\text { Therapy }\end{array}$ & $\begin{array}{l}\text { Non-Voxel } \\
\text { Broad } \\
\text { Beam }\end{array}$ & Yes & 18.0 & 20.7 & $87 \%$ \\
\hline 6 & BL Novalis Tx & 6MV SRS & $8 \mathrm{FF}$ & $\mathrm{NC}$ & Pinnacle & Convolution & Yes & 18.0 & 22.4 & $80 \%$ \\
\hline 7 & ELK Agility & $6 \mathrm{MV}$ & 3DCA & $\mathrm{NC}$ & Monaco & MC Photon & Yes & 18.0 & 19.9 & $90 \%$ \\
\hline 8 & BL Novalis Tx & 6MV SRS & $4 \mathrm{DCA}$ & NC & BL iPlan & $\begin{array}{l}\text { Pencil } \\
\text { Beam }\end{array}$ & Yes & 18.0 & 23.1 & $78 \%$ \\
\hline 9 & $\begin{array}{c}\text { VRN } \\
\text { TrueBeam }\end{array}$ & $6 \mathrm{MV}$ & 4DCA & $\mathrm{NC}$ & BL iPlan & $\begin{array}{l}\text { Pencil } \\
\text { Beam }\end{array}$ & Yes & 18.0 & 22.8 & $79 \%$ \\
\hline 10 & $\begin{array}{c}\text { ELK } \\
\text { VersaHD }\end{array}$ & $6 \mathrm{MV}$ & 5VMAT & $\mathrm{NC}$ & Monaco & MC Photon & Yes & 21.0 & 26.3 & $80 \%$ \\
\hline 11 & $\begin{array}{c}\text { VRN } \\
\text { TrueBeam }\end{array}$ & 10MV FFF & 2VMAT & C & Eclipse & AAA & Yes & 20.0 & 23.3 & $86 \%$ \\
\hline 12 & BL Novalis Tx & 6MV SRS & 4DCA & $\mathrm{NC}$ & BL iPlan & $\begin{array}{l}\text { Pencil } \\
\text { Beam }\end{array}$ & Yes & 18.0 & 23.2 & $78 \%$ \\
\hline 13 & $\begin{array}{c}\text { VRN } \\
\text { TrueBeam }\end{array}$ & $6 \mathrm{MV}$ FFF & 1VMAT & C & Eclipse & AAA & Yes & 16.0 & 19.9 & $80 \%$ \\
\hline 14 & $\begin{array}{c}\text { VRN } \\
\text { TrueBeam }\end{array}$ & 10MV FFF & 1VMAT & $\mathrm{C}$ & Eclipse & AAA & Yes & 16.0 & 20.3 & $79 \%$ \\
\hline 15 & $\begin{array}{c}\text { ELK } \\
\text { BeamMod }\end{array}$ & $6 \mathrm{MV}$ & $8 \mathrm{FF}$ & $\mathrm{NC}$ & Pinnacle & Convolution & Yes & 21.0 & 23.5 & $89 \%$ \\
\hline 16 & $\begin{array}{l}\text { VRN } \\
\text { TrueBeam } \\
\text { STx }\end{array}$ & $6 \mathrm{MV}$ & $5 \mathrm{DCA}$ & $\mathrm{NC}$ & BL iPlan & $\begin{array}{l}\text { Pencil } \\
\text { Beam }\end{array}$ & Yes & 18.0 & 22.7 & $79 \%$ \\
\hline 17 & $\begin{array}{c}\text { ELK } \\
\text { VersaHD }\end{array}$ & $6 \mathrm{MV}$ FFF & 3VMAT & $\mathrm{NC}$ & Monaco & MC Photon & Yes & 18.0 & 34.8 & $52 \%$ \\
\hline 18 & $\begin{array}{c}\text { VRN } \\
\text { TrueBeam }\end{array}$ & 10MV FFF & 4VMAT & $\mathrm{NC}$ & Eclipse & Acuros & Yes & 20.0 & 30.0 & $67 \%$ \\
\hline 19 & $\begin{array}{c}\text { ELK } \\
\text { BeamMod }\end{array}$ & $6 \mathrm{MV}$ & 7DCA & $\mathrm{NC}$ & Pinnacle & Convolution & Yes & 18.0 & 24.1 & $75 \%$ \\
\hline 20 & $\begin{array}{c}\text { VRN } \\
\text { TrueBeam } \\
\text { STx }\end{array}$ & $6 \mathrm{MV}$ & 5DCA & $\mathrm{NC}$ & BL iPlan & $\begin{array}{l}\text { Pencil } \\
\text { Beam }\end{array}$ & Yes & 21.0 & 27.1 & $77 \%$ \\
\hline 21 & VRN ix2100 & $6 \mathrm{MV}$ & 7VMAT & $\mathrm{NC}$ & Eclipse & AAA & Yes & 21.0 & 30.5 & $69 \%$ \\
\hline 22 & $\begin{array}{l}\text { VRN } \\
\text { TrueBeam } \\
\text { STx } \\
\end{array}$ & $6 \mathrm{MV}$ FFF & $5 \mathrm{DCA}$ & $\mathrm{NC}$ & BL iPlan & $\begin{array}{l}\text { Pencil } \\
\text { Beam }\end{array}$ & Yes & 18.0 & 22.2 & $81 \%$ \\
\hline 23 & $\begin{array}{c}\text { ELK GK } \\
\text { Perfexion }\end{array}$ & ${ }^{60} \mathrm{Co}$ & 17 shots & $\mathrm{NC}$ & $\begin{array}{c}\text { Gamma } \\
\text { Plan }\end{array}$ & TMR10 & No & 18.0 & 40.9 & $44 \%$ \\
\hline 24 & $\begin{array}{c}\text { ELK GK } \\
\text { Perfexion }\end{array}$ & ${ }^{60} \mathrm{Co}$ & 19 shots & $\mathrm{NC}$ & $\begin{array}{c}\text { Gamma } \\
\text { Plan }\end{array}$ & TMR10 & No & 20.0 & 40.0 & $50 \%$ \\
\hline 25 & ELK GK Icon & ${ }^{60} \mathrm{Co}$ & 20 shots & $\mathrm{NC}$ & $\begin{array}{c}\text { Gamma } \\
\text { Plan }\end{array}$ & TMR10 & No & 18.0 & 36.7 & $49 \%$ \\
\hline 26 & ELK GK Icon & ${ }^{60} \mathrm{Co}$ & 22 shots & $\mathrm{NC}$ & $\begin{array}{c}\text { Gamma } \\
\text { Plan }\end{array}$ & TMR10 & No & 18.0 & 36.0 & $50 \%$ \\
\hline 27 & $\begin{array}{l}\text { ELK GK } \\
\text { Perfexion }\end{array}$ & ${ }^{60} \mathrm{Co}$ & 11 shots & $\mathrm{NC}$ & $\begin{array}{c}\text { Gamma } \\
\text { Plan }\end{array}$ & TMR10 & No & 18.0 & 39.1 & $46 \%$ \\
\hline 28 & $\begin{array}{l}\text { ELK GK } \\
\text { Perfexion }\end{array}$ & ${ }^{60} \mathrm{Co}$ & 22 shots & $\mathrm{NC}$ & $\begin{array}{c}\text { Gamma } \\
\text { Plan }\end{array}$ & TMR10 & No & 18.0 & 40.9 & $44 \%$ \\
\hline 29 & $\begin{array}{c}\text { ELK GK } \\
\text { Perfexion }\end{array}$ & ${ }^{60} \mathrm{Co}$ & 22 shots & $\mathrm{NC}$ & $\begin{array}{c}\text { Gamma } \\
\text { Plan }\end{array}$ & TMR10 & No & 18.0 & 41.4 & $43 \%$ \\
\hline 30 & ACC CK VSI & $6 \mathrm{MV}$ FFF & 138 beams & $\mathrm{NC}$ & $\begin{array}{l}\text { Multi } \\
\text { Plan }\end{array}$ & Ray Tracing & Yes & 21.0 & 32.3 & $65 \%$ \\
\hline 31 & ACC CK VSI & $6 \mathrm{MV}$ FFF & 123 beams & $\mathrm{NC}$ & $\begin{array}{l}\text { Multi } \\
\text { Plan }\end{array}$ & Ray Tracing & Yes & 18.0 & 25.7 & $70 \%$ \\
\hline 32 & ACC CK VSI & $6 \mathrm{MV}$ FFF & 139 beams & $\mathrm{NC}$ & $\begin{array}{l}\text { Multi } \\
\text { Plan }\end{array}$ & Ray Tracing & Yes & 18.0 & 34.0 & $53 \%$ \\
\hline 33 & ACC CK VSI & $6 \mathrm{MV}$ FFF & 109 beams & $\mathrm{NC}$ & $\begin{array}{l}\text { Multi } \\
\text { Plan }\end{array}$ & Ray Tracing & Yes & 20.0 & 30.8 & $65 \%$ \\
\hline
\end{tabular}

Table 1: Summary of equipment, techniques and prescription practices of the audit participants. The centres are grouped by platform and in random order, different to the order shown in the results, to avoid identification of individual centres. On-board imaging for positioning the phantom was used by all participants except centres 3, 23, 24, 27, 28 and 29. (VRN=Varian, BL=Brainlab, ELK=Elekta,

$\mathrm{ACC}=$ Accuray, $\mathrm{GK}=$ Gamma Knife, $\mathrm{CK}=$ Cyberknife (with cones), DCA=Dynamic Conformal Arcs,

FF=Fixed Fields, CCA=Circular Collimator Arcs, VMAT=Volumetric Modulated Arc Therapy) 


\section{Pre- assessment}

1. A high resolution DICOM image set of the phantom was send to the host centre with instructions for contouring the target and the brainstem.

2. The host centre outlined the structures and reported back to auditors.

3. After approval from the auditors, the host centre practised on the planning scenario available and prepared a plan prior to the day of the audit.

\section{On day of assessment}

1. The phantom was presented to the host centre with dummy film and alanine pellets in place.

2. The immobilisation device (frame/mask) was applied to the phantom followed by a CTscan in stereotactic conditions following the local SRS protocol.

3. The acquired scan was fused/co-registered with the previously sent image set and the alanine pellets were delineated.

4. The plan was recalculated on the CT scan acquired on the day and exported to the treatment platform for delivery.

5. Output measurements in reference conditions were performed by both the auditor and auditee.

6. The dummy film and alanine pellets were replaced with real film and alanine pellets.

7. The audit phantom was accurately positioned on the treatment platform, with/without the use of on-board imaging, and the plan was delivered.

\section{Post- assessment}

1. The participating centre provided the auditors with RTDOSE DICOM files and dose-volume histograms from the delivered treatment plan for comparison to the measured alanine and films.

2. Alanine pellets and films were returned to the responsible laboratories for analysis

3. Alanine and film measured doses were compared to the TPS predicted doses.

4. The results were checked by a second person and a provisional report with the results was prepared and sent to the participating centre.

Figure 2: Flowchart diagram showing the main steps of the procedure followed in performing 568 the end-to-end assessments. 
Short title: Multi-institutional audit of dosimetric delivery in radiosurgery

1

2

3

4

5

6

7

8

9

10

11

12

13

14

15

16

17

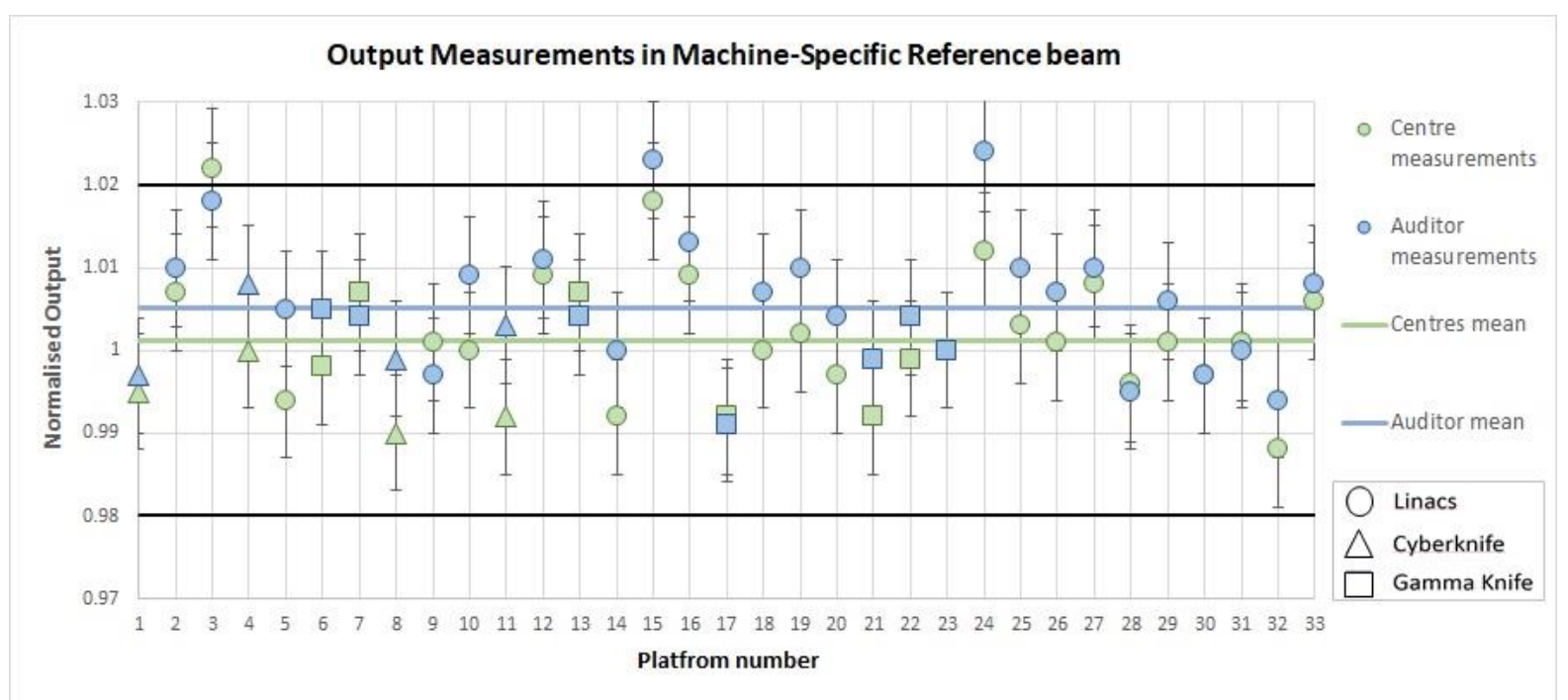

Figure 3: Output measurements in local reference conditions for the 33 platforms that participated in the audit. An uncertainty of $\pm 0.7 \%$ ( $k=1)$ is indicated by the error bars as a standard protocol based on the calibration certificate of the detector. The "acceptable" tolerances of $\pm 2 \%$ are indicated. 
Short title: Multi-institutional audit of dosimetric delivery in radiosurgery

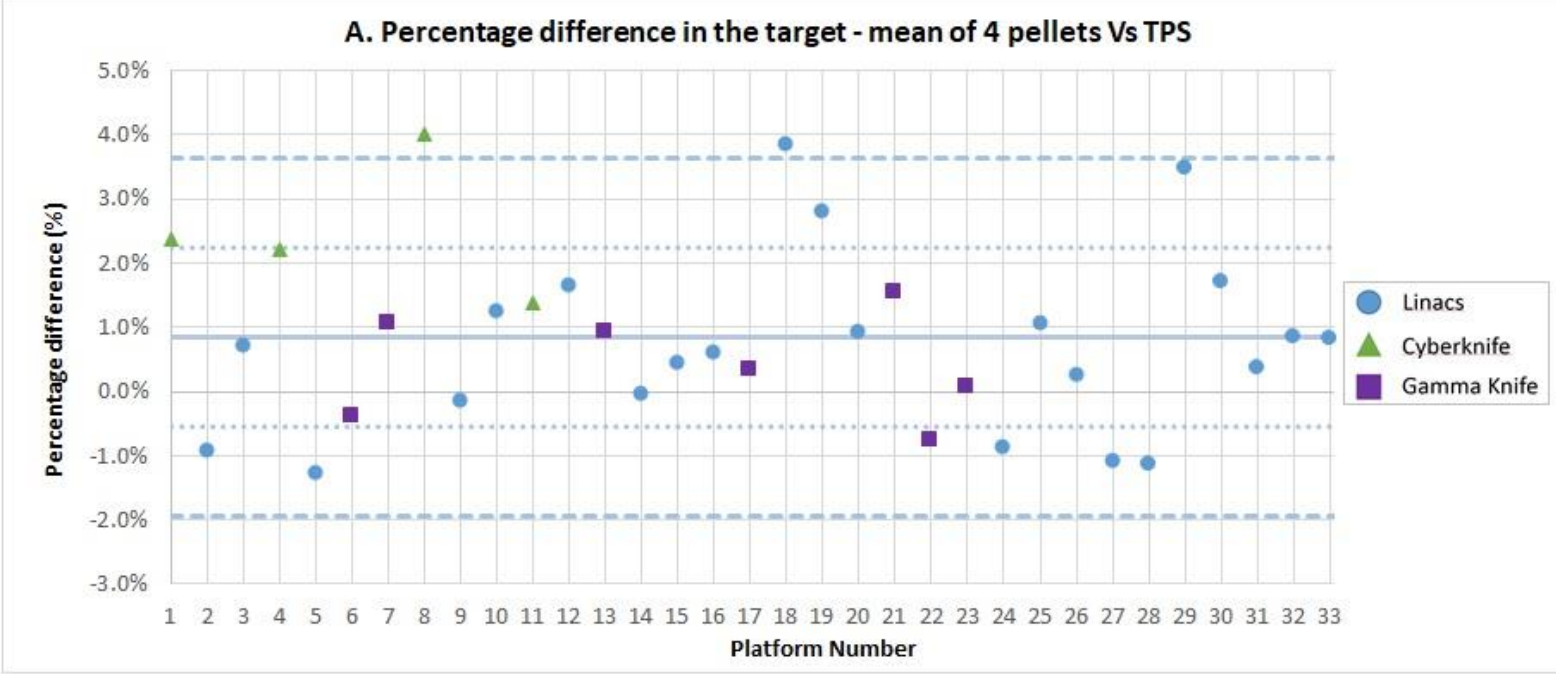

B. Percentage difference in the brainstem - mean of 4 alanine pellets Vs TPS (normalised to $12 \mathrm{~Gy}$-nominal brainstem tolerance dose)

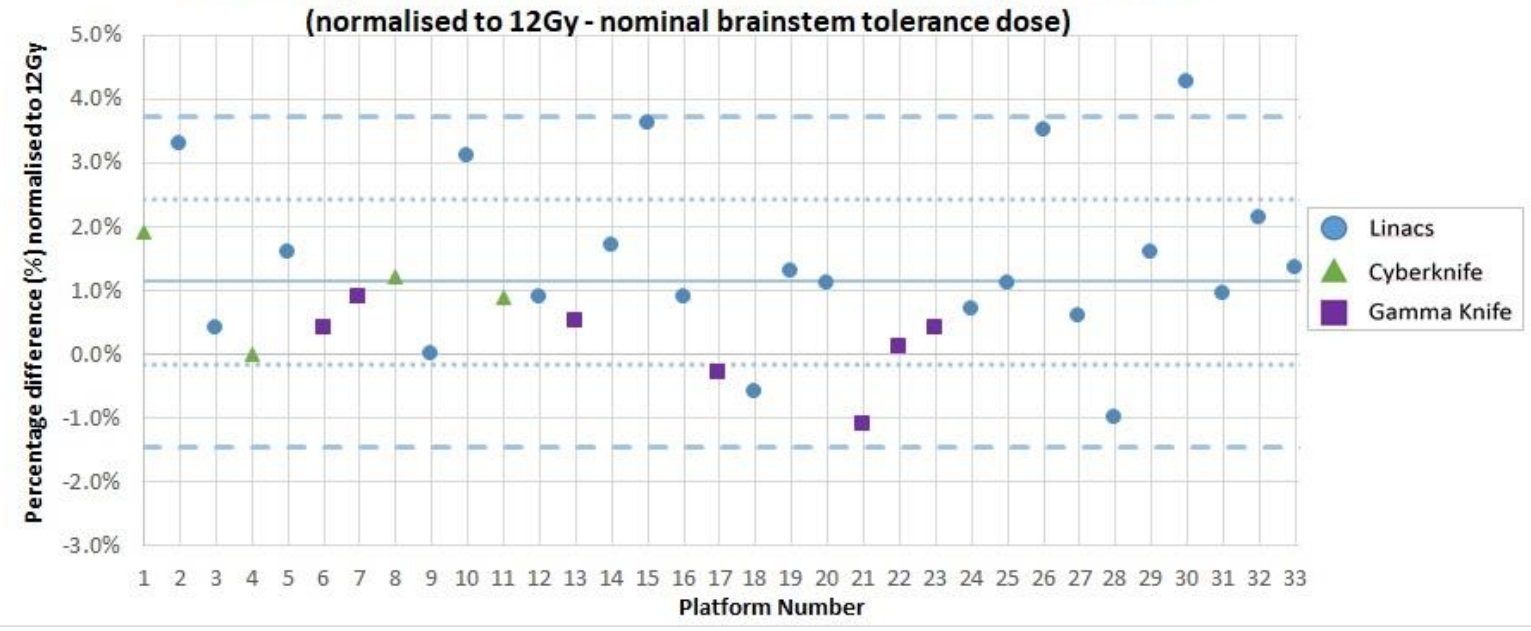

Figure 4: Alanine pellet measurements performed in the target (4A) and organ at risk (4B). Platform groups are indicated in the legends. The mean for all centres is represented by the solid blue line, the dotted lines represent one standard deviation of the mean and the dashed lines represent two standard deviations of the mean. 

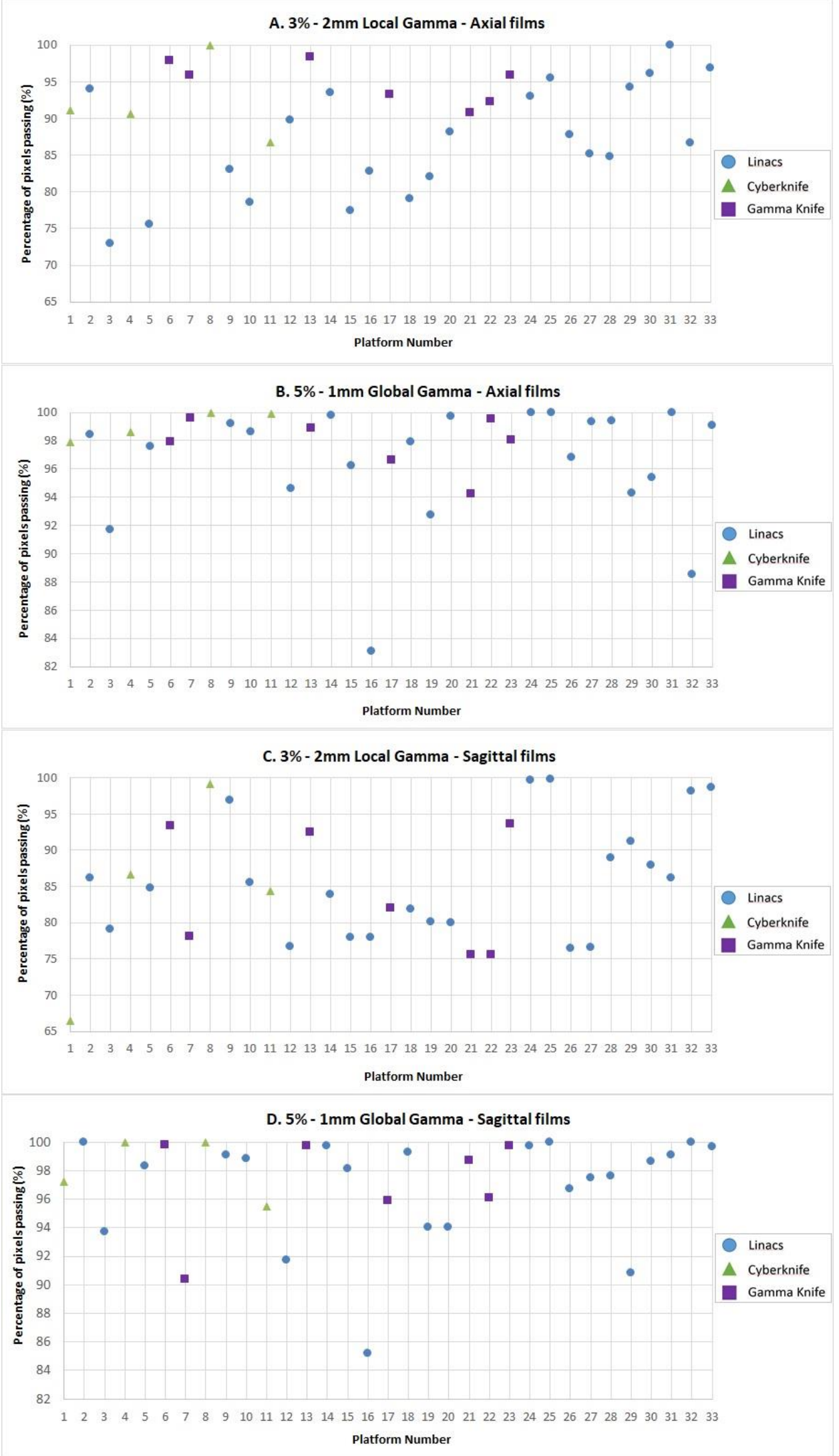

Figure 5: Axial (5A\&5B) and Sagittal (5C\&5D) film passing rates of the local gamma criterion of 3\% $2 \mathrm{~mm}$ and the global gamma criterion of $5 \%-1 \mathrm{~mm}$ for the 33 platforms that were assessed. 
Short title: Multi-institutional audit of dosimetric delivery in radiosurgery

1

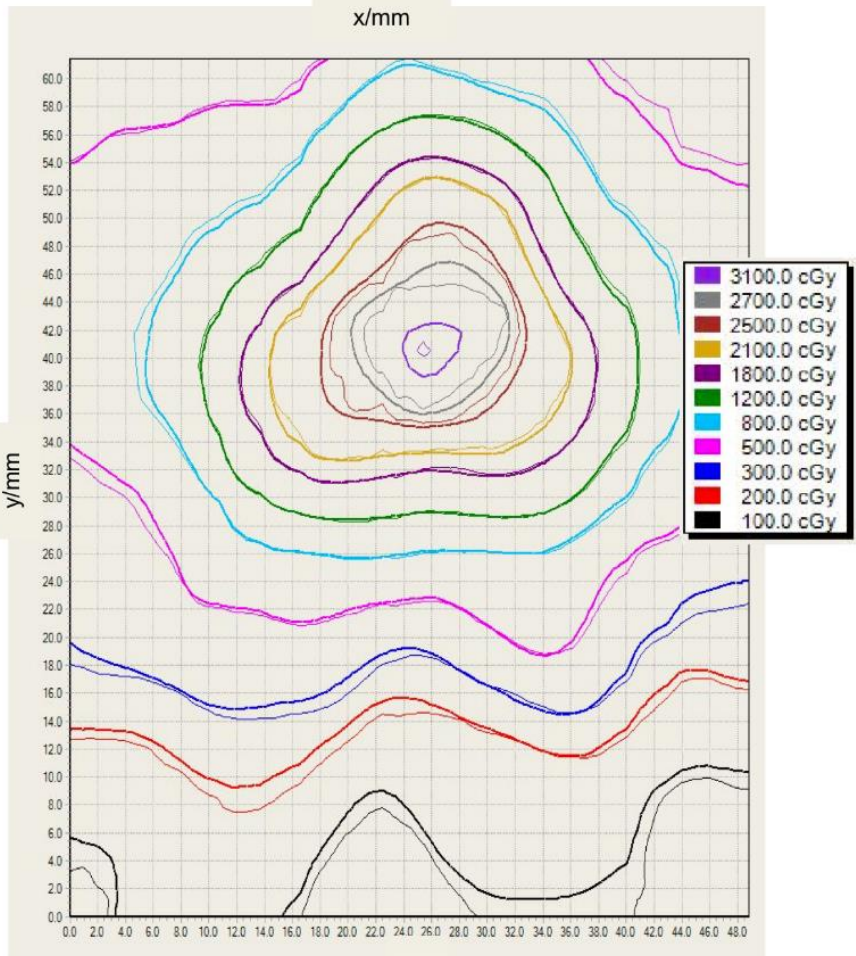

Axial

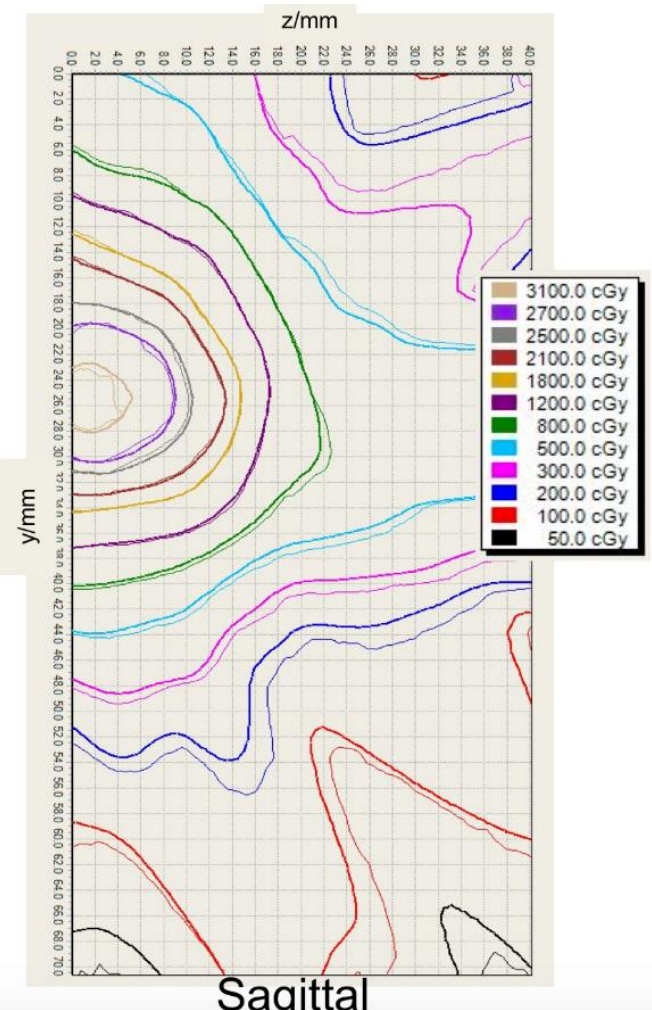

Sagittal

Supplementary Figure 1: Example of dose distribution comparisons between the film-measured doses (thin lines) and the treatment planning system- calculated doses (thick lines) for the axial and the sagittal films used. 

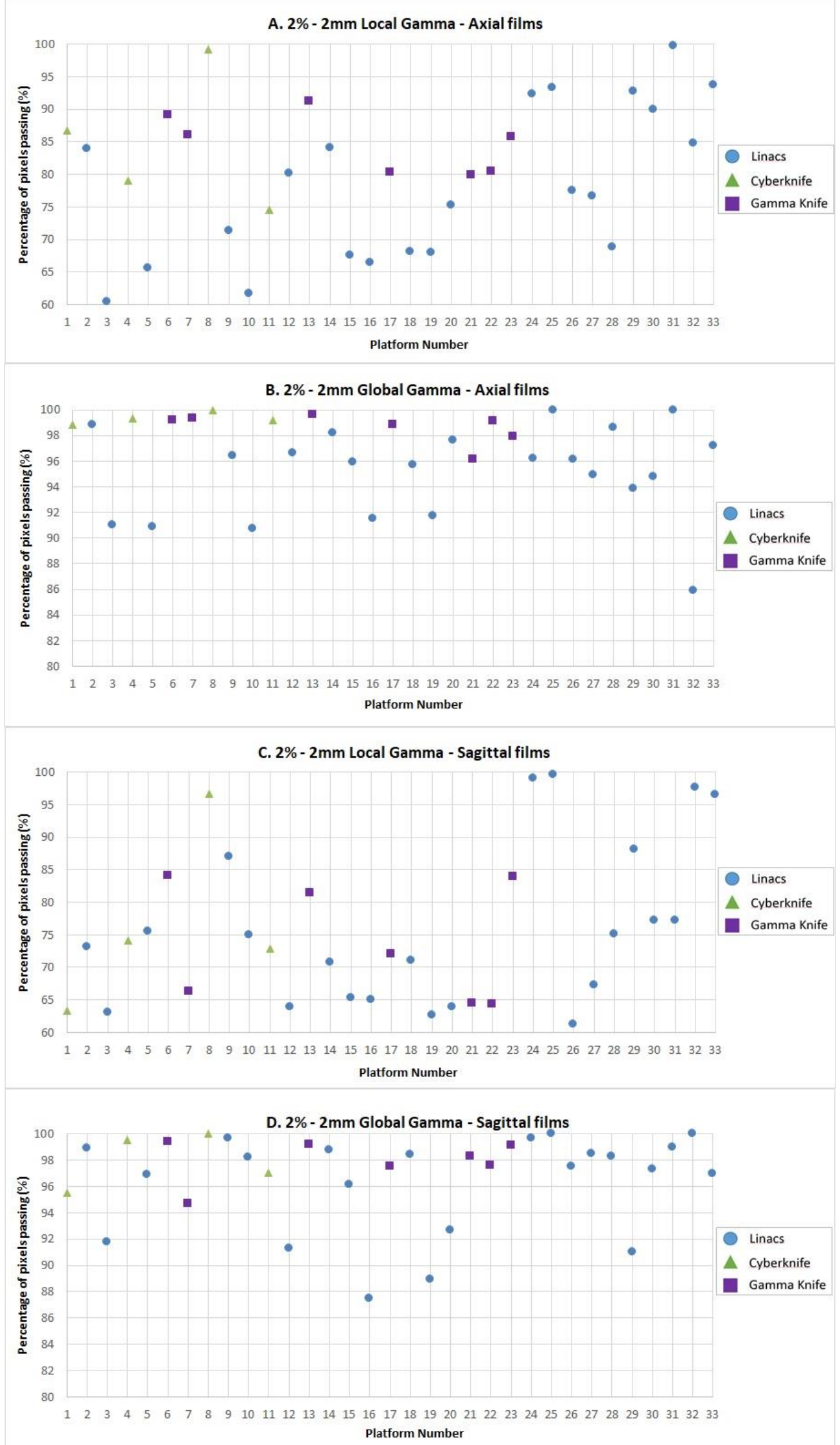

645 Supplementary Figure 2: Axial (A\&B) and Sagittal (C\&D) film passing rates of the local gamma and 647 the global gamma criteria of $2 \%-2 \mathrm{~mm}$ the 33 platforms that were assessed. 


\begin{tabular}{|c|c|c|c|c|c|c|c|c|c|c|}
\hline $\begin{array}{l}\text { Platform } \\
\text { number }\end{array}$ & Platform & Energy & Technique & $\begin{array}{l}\text { Coplanar } \\
\text { / Non- } \\
\text { coplanar }\end{array}$ & TPS & $\begin{array}{c}\text { Dose } \\
\text { Calculation } \\
\text { Algorithm }\end{array}$ & $\begin{array}{l}\text { Density } \\
\text { Heterog. } \\
\text { Correction }\end{array}$ & $\begin{array}{l}\text { Peripheral } \\
\text { Prescr. } \\
\text { Dose (Gy) }\end{array}$ & $\begin{array}{l}\text { Max } \\
\text { Dose } \\
\text { (Gy) }\end{array}$ & $\begin{array}{c}\text { Pr Iso. } \\
\text { Relative to } \\
\text { Dmax (\%) }\end{array}$ \\
\hline 1 & $\begin{array}{l}\text { VRN } \\
\text { TrueBeam } \\
\text { STx }\end{array}$ & 10MV FFF & 4DCA & $\mathrm{NC}$ & BL iPlan & $\begin{array}{l}\text { Pencil } \\
\text { Beam }\end{array}$ & Yes & 21.0 & 26.4 & $80 \%$ \\
\hline 2 & BL Novalis Tx & $6 \mathrm{MV}$ & 9FF & $\mathrm{NC}$ & BL iPlan & $\begin{array}{l}\text { Pencil } \\
\text { Beam }\end{array}$ & Yes & 17.5 & 22.7 & $77 \%$ \\
\hline 3 & VRN 2100x & $6 \mathrm{MV}$ & 4CCA & $\mathrm{NC}$ & BL iPlan & $\begin{array}{l}\text { Circular } \\
\text { Cone }\end{array}$ & Yes & 18.0 & 23.6 & $76 \%$ \\
\hline 4 & ELK Synergy & $6 \mathrm{MV}$ & 5VMAT & $\mathrm{NC}$ & Pinnacle & Convolution & Yes & 18.0 & 22.9 & $79 \%$ \\
\hline 5 & $\begin{array}{c}\text { ACC } \\
\text { Tomotherapy }\end{array}$ & 6MV FFF & $\begin{array}{l}\text { Tomo } \\
\text { Therapy }\end{array}$ & $\mathrm{C}$ & $\begin{array}{l}\text { Tomo } \\
\text { Therapy }\end{array}$ & $\begin{array}{l}\text { Non-Voxel } \\
\text { Broad } \\
\text { Beam }\end{array}$ & Yes & 18.0 & 20.7 & $87 \%$ \\
\hline 6 & BL Novalis Tx & 6MV SRS & $8 \mathrm{FF}$ & $\mathrm{NC}$ & Pinnacle & Convolution & Yes & 18.0 & 22.4 & $80 \%$ \\
\hline 7 & ELK Agility & $6 \mathrm{MV}$ & 3DCA & $\mathrm{NC}$ & Monaco & MC Photon & Yes & 18.0 & 19.9 & $90 \%$ \\
\hline 8 & BL Novalis Tx & 6MV SRS & 4DCA & $\mathrm{NC}$ & BL iPlan & $\begin{array}{l}\text { Pencil } \\
\text { Beam }\end{array}$ & Yes & 18.0 & 23.1 & $78 \%$ \\
\hline 9 & $\begin{array}{c}\text { VRN } \\
\text { TrueBeam }\end{array}$ & $6 \mathrm{MV}$ & 4DCA & NC & BL iPlan & $\begin{array}{l}\text { Pencil } \\
\text { Beam }\end{array}$ & Yes & 18.0 & 22.8 & $79 \%$ \\
\hline 10 & $\begin{array}{c}\text { ELK } \\
\text { VersaHD }\end{array}$ & $6 \mathrm{MV}$ & 5VMAT & $\mathrm{NC}$ & Monaco & MC Photon & Yes & 21.0 & 26.3 & $80 \%$ \\
\hline 11 & $\begin{array}{c}\text { VRN } \\
\text { TrueBeam }\end{array}$ & 10MV FFF & 2VMAT & $\mathrm{C}$ & Eclipse & AAA & Yes & 20.0 & 23.3 & $86 \%$ \\
\hline 12 & BL Novalis Tx & 6MV SRS & 4DCA & $\mathrm{NC}$ & BL iPlan & $\begin{array}{l}\text { Pencil } \\
\text { Beam }\end{array}$ & Yes & 18.0 & 23.2 & $78 \%$ \\
\hline 13 & $\begin{array}{c}\text { VRN } \\
\text { TrueBeam }\end{array}$ & 6MV FFF & 1VMAT & $\mathrm{C}$ & Eclipse & AAA & Yes & 16.0 & 19.9 & $80 \%$ \\
\hline 14 & $\begin{array}{c}\text { VRN } \\
\text { TrueBeam }\end{array}$ & 10MV FFF & 1VMAT & $\mathrm{C}$ & Eclipse & AAA & Yes & 16.0 & 20.3 & $79 \%$ \\
\hline 15 & $\begin{array}{c}\text { ELK } \\
\text { BeamMod }\end{array}$ & $6 \mathrm{MV}$ & $8 \mathrm{FF}$ & NC & Pinnacle & Convolution & Yes & 21.0 & 23.5 & $89 \%$ \\
\hline 16 & $\begin{array}{c}\text { VRN } \\
\text { TrueBeam } \\
\text { STx } \\
\end{array}$ & $6 \mathrm{MV}$ & $5 D C A$ & NC & BL iPlan & $\begin{array}{l}\text { Pencil } \\
\text { Beam }\end{array}$ & Yes & 18.0 & 22.7 & $79 \%$ \\
\hline 17 & $\begin{array}{c}\text { ELK } \\
\text { VersaHD }\end{array}$ & 6MV FFF & 3VMAT & NC & Monaco & MC Photon & Yes & 18.0 & 34.8 & $52 \%$ \\
\hline 18 & $\begin{array}{c}\text { VRN } \\
\text { TrueBeam }\end{array}$ & 10MV FFF & 4VMAT & NC & Eclipse & Acuros & Yes & 20.0 & 30.0 & $67 \%$ \\
\hline 19 & $\begin{array}{c}\text { ELK } \\
\text { BeamMod }\end{array}$ & $6 \mathrm{MV}$ & 7DCA & NC & Pinnacle & Convolution & Yes & 18.0 & 24.1 & $75 \%$ \\
\hline 20 & $\begin{array}{c}\text { VRN } \\
\text { TrueBeam } \\
\text { STx }\end{array}$ & $6 \mathrm{MV}$ & $5 D C A$ & $\mathrm{NC}$ & BL iPlan & $\begin{array}{l}\text { Pencil } \\
\text { Beam }\end{array}$ & Yes & 21.0 & 27.1 & $77 \%$ \\
\hline 21 & VRN ix2100 & $6 \mathrm{MV}$ & 7VMAT & $\mathrm{NC}$ & Eclipse & AAA & Yes & 21.0 & 30.5 & $69 \%$ \\
\hline 22 & $\begin{array}{l}\text { VRN } \\
\text { TrueBeam } \\
\text { STx }\end{array}$ & $6 \mathrm{MV}$ FFF & $5 D C A$ & NC & BL iPlan & $\begin{array}{l}\text { Pencil } \\
\text { Beam }\end{array}$ & Yes & 18.0 & 22.2 & $81 \%$ \\
\hline 23 & $\begin{array}{c}\text { ELK GK } \\
\text { Perfexion }\end{array}$ & ${ }^{60} \mathrm{Co}$ & 17 shots & $\mathrm{NC}$ & $\begin{array}{c}\text { Gamma } \\
\text { Plan }\end{array}$ & TMR10 & No & 18.0 & 40.9 & $44 \%$ \\
\hline 24 & $\begin{array}{c}\text { ELK GK } \\
\text { Perfexion }\end{array}$ & ${ }^{60} \mathrm{Co}$ & 19 shots & $\mathrm{NC}$ & $\begin{array}{c}\text { Gamma } \\
\text { Plan }\end{array}$ & TMR10 & No & 20.0 & 40.0 & $50 \%$ \\
\hline 25 & ELK GK Icon & ${ }^{60} \mathrm{Co}$ & 20 shots & NC & $\begin{array}{c}\text { Gamma } \\
\text { Plan }\end{array}$ & TMR10 & No & 18.0 & 36.7 & $49 \%$ \\
\hline 26 & ELK GK Icon & ${ }^{60} \mathrm{Co}$ & 22 shots & $\mathrm{NC}$ & $\begin{array}{c}\text { Gamma } \\
\text { Plan }\end{array}$ & TMR10 & No & 18.0 & 36.0 & $50 \%$ \\
\hline 27 & $\begin{array}{c}\text { ELK GK } \\
\text { Perfexion }\end{array}$ & ${ }^{60} \mathrm{Co}$ & 11 shots & $\mathrm{NC}$ & $\begin{array}{c}\text { Gamma } \\
\text { Plan }\end{array}$ & TMR10 & No & 18.0 & 39.1 & $46 \%$ \\
\hline 28 & $\begin{array}{c}\text { ELK GK } \\
\text { Perfexion }\end{array}$ & ${ }^{60} \mathrm{Co}$ & 22 shots & $\mathrm{NC}$ & $\begin{array}{c}\text { Gamma } \\
\text { Plan }\end{array}$ & TMR10 & No & 18.0 & 40.9 & $44 \%$ \\
\hline 29 & $\begin{array}{c}\text { ELK GK } \\
\text { Perfexion }\end{array}$ & ${ }^{60} \mathrm{Co}$ & 22 shots & $\mathrm{NC}$ & $\begin{array}{c}\text { Gamma } \\
\text { Plan }\end{array}$ & TMR10 & No & 18.0 & 41.4 & $43 \%$ \\
\hline 30 & ACC CK VSI & $6 \mathrm{MV}$ FFF & 138 beams & NC & $\begin{array}{l}\text { Multi } \\
\text { Plan }\end{array}$ & Ray Tracing & Yes & 21.0 & 32.3 & $65 \%$ \\
\hline 31 & ACC CK VSI & $6 \mathrm{MV}$ FFF & 123 beams & $\mathrm{NC}$ & $\begin{array}{l}\text { Multi } \\
\text { Plan }\end{array}$ & Ray Tracing & Yes & 18.0 & 25.7 & $70 \%$ \\
\hline 32 & ACC CK VSI & 6MV FFF & 139 beams & $\mathrm{NC}$ & $\begin{array}{l}\text { Multi } \\
\text { Plan }\end{array}$ & Ray Tracing & Yes & 18.0 & 34.0 & $53 \%$ \\
\hline 33 & ACC CK VSI & $6 \mathrm{MV}$ FFF & 109 beams & $\mathrm{NC}$ & $\begin{array}{l}\text { Multi } \\
\text { Plan }\end{array}$ & Ray Tracing & Yes & 20.0 & 30.8 & $65 \%$ \\
\hline
\end{tabular}

Table 1: Summary of equipment, techniques and prescription practices of the audit participants. The centres are grouped by platform and in random order, different to the order shown in the results, to avoid identification of individual centres. On-board imaging for positioning the phantom was used by all participants except centres 3, 23, 24, 27, 28 and 29. (VRN=Varian, BL=Brainlab, ELK=Elekta,

$A C C=A c c u r a y, G K=G a m m a$ Knife, $C K=$ Cyberknife, $D C A=$ Dynamic Conformal Arcs, FF=Fixed Fields, $\mathrm{CCA}=$ Circular Collimator Arcs, VMAT=Volumetric Modulated Arc Therapy) 


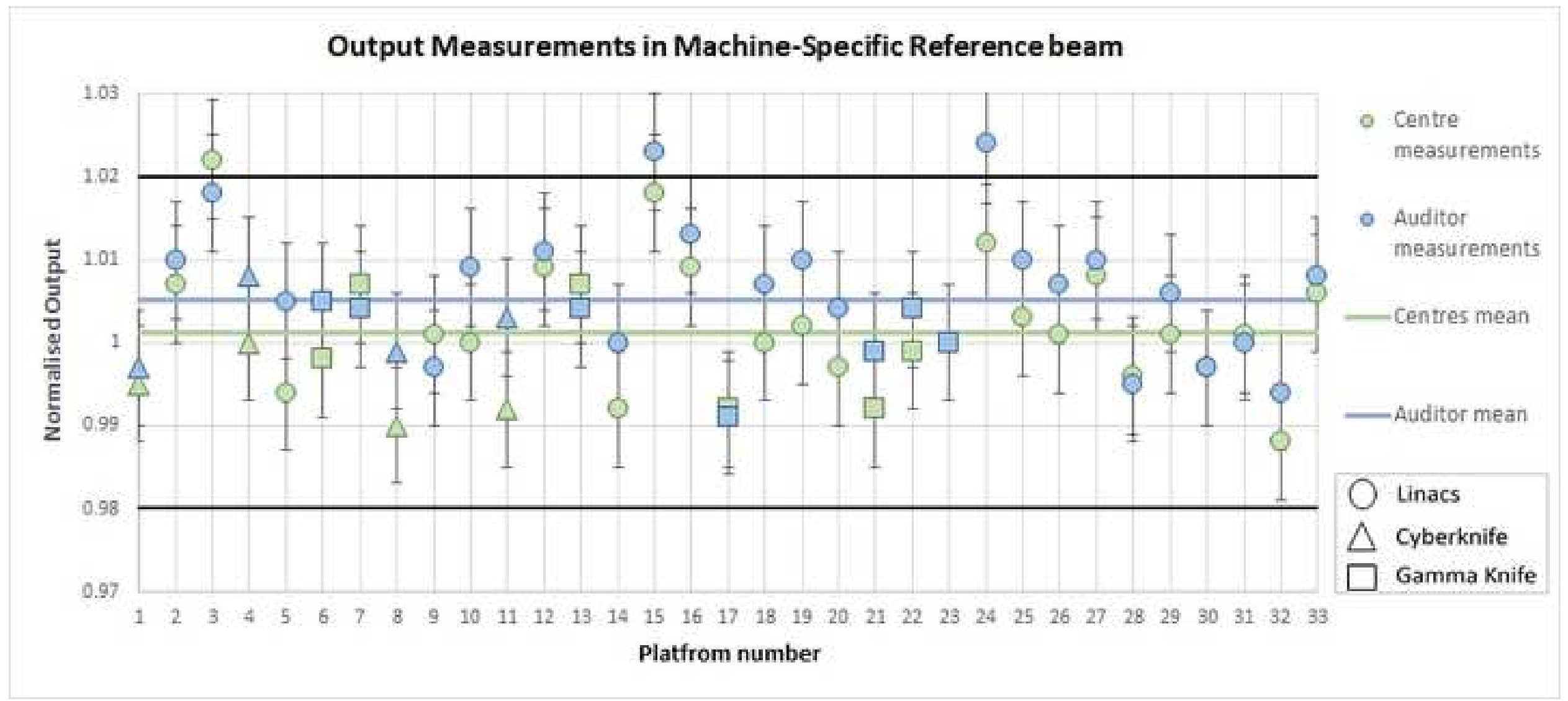

Auditor

measurements

- Centres mean

Cyberknife

Gamma Knife

\section{Platfrom number}




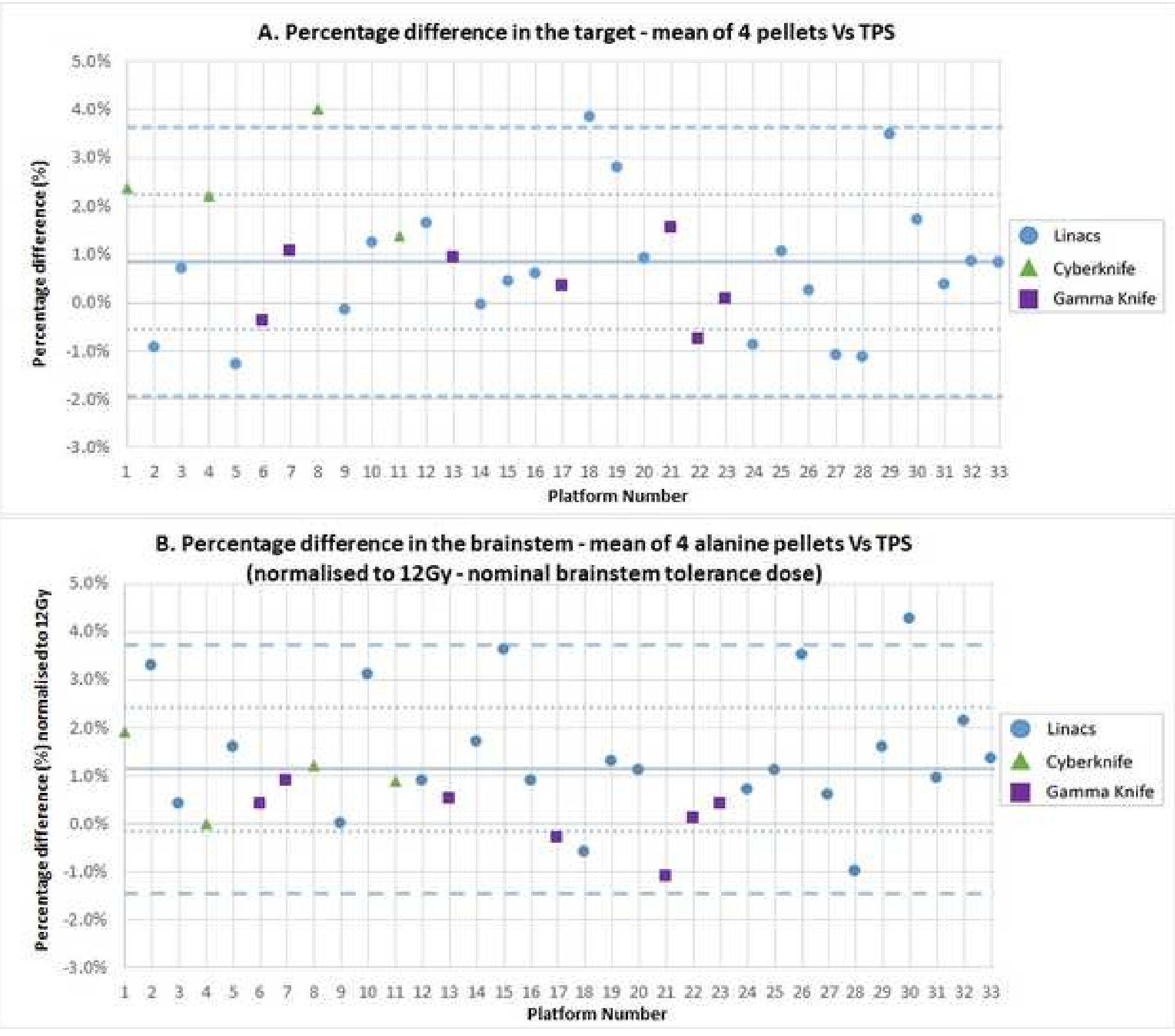

A. Percentage difference in the target - mean of 4 pellets Vs TPS 
A. 3\%- $2 \mathrm{~mm}$ Local Gamma - Axial films
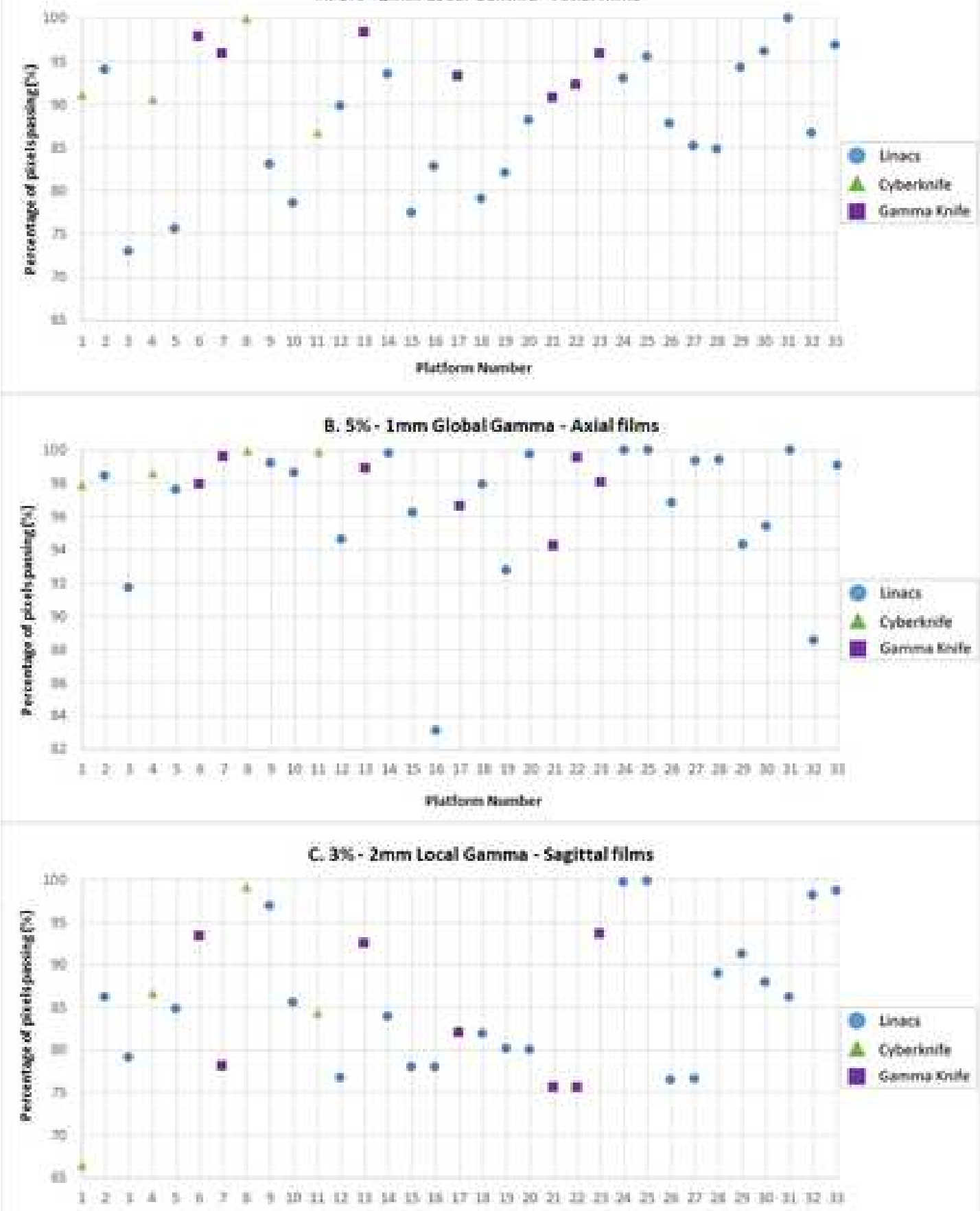

Mutsorm Number

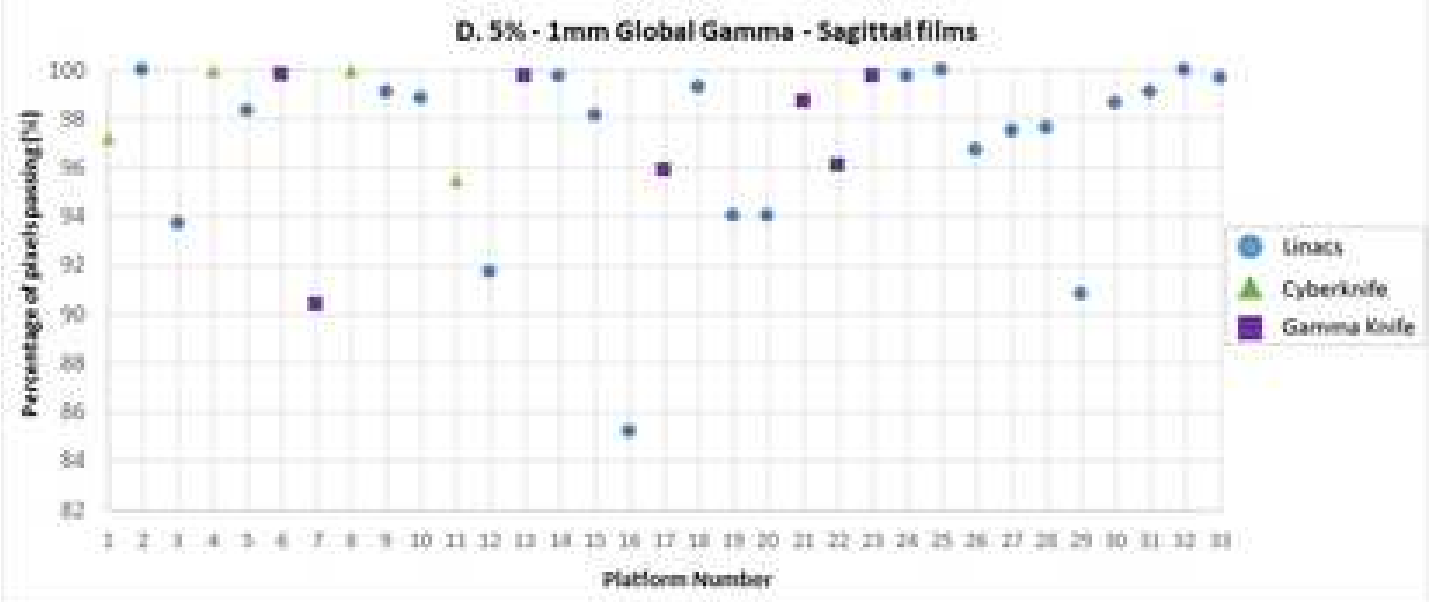


Click here to access/download Supplementary Files SF2.Resubmission_Supplementary_Figure_2_edited_20 20.jpg 
$08 / 12 / 2019$

Dear Editor,

I hereby confirm that none of the authors of the submitted manuscript have a conflict of interest to declare.

Regards,

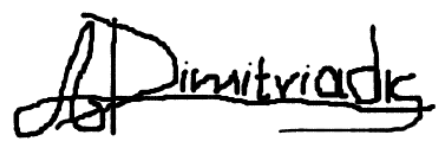

\section{Alexis Dimitriadis}

$\mathrm{BSc}, \mathrm{MSc}, \mathrm{PhD}$

"Multi-institutional dosimetric delivery assessment of intracranial stereotactic radiosurgery on different treatment platforms"

Authors: Alexis Dimitriadis ${ }^{1,2} \mathrm{PhD}^{*}$, Yatman Tsang ${ }^{4} \mathrm{PhD}$, Russell A.S. Thomas ${ }^{2} \mathrm{MSc}$, Antony L. Palmer ${ }^{1,3} \mathrm{PhD}$, David Eaton ${ }^{4} \mathrm{PhD}$, Jonathan Lee ${ }^{4} \mathrm{MSc}$, Rushil Patel ${ }^{4} \mathrm{MSc}$, lleana Silvestre Patallo ${ }^{2}$ MSc, Clare Gouldstone ${ }^{2}$ MSc, Julia A.D. Snaith ${ }^{2}$ MSc, Karen J. Kirkby ${ }^{6,7} \mathrm{PhD}$, Andrew Nisbet ${ }^{2,8} \mathrm{PhD}$, Catharine H. Clark ${ }^{1,2,4,5} \mathrm{PhD}$

1. Faculty of Engineering and Physical Sciences, University of Surrey, Guildford, Surrey, UK

2. Radiation Dosimetry Group, National Physical Laboratory, Teddington, Middlesex, UK

3. Medical Physics Department, Portsmouth Hospitals NHS Trust, Portsmouth, UK

4. Radiotherapy Trials Quality Assurance Group, Mount Vernon Hospital, London, UK

5. Department of Medical Physics, Royal Surrey County Hospital NHS Foundation Trust, Guildford, Surrey, UK

6. The Christie NHS Foundation Trust, Manchester, UK

7. Institute of Cancer Sciences, University of Manchester, Manchester, UK

8. University College London, London, UK

*Corresponding author: dimitriadis.alexis@gmail.com 